XII.

\title{
Bericht über die vom 1. April 1881 bis 1. April 1884 in der otiatrischen Universitätsklinik zn Berlin behandelten Ohrenkranken.
}

\author{
Yon \\ Dr. I. Jacobson, \\ Assistent.
}

Die folgenden Mittheilungen beziehen sich anf die Thätigkeit der unter Leitung des Herrn Prof. Lucae stehenden Berliner Universitätsklinik für Ohrenkranke während der ersten drei Jahre ihres Bestehens. Dieselbe verfügte in der angegebenen Zeit über 11 Betten, zu welchen im April 1884 noch fernere 9 hinzugekommen sind. Die Zahl der aufgenommenen Kranken betrug 232; unter diesen waren 104 Männer, $66 \mathrm{~W}$ eiber und 62 Kinder unter 15 Jahren, worunter 31 Knaben und 31 Mädchen. Es wurden 38 geheilt, 137 gebessert, 42 ungeheilt entlassen, 3 starben, 9 wurden in das laufende Jahr übernommen, und 3 in die chirurgische Klinik verlegt, und zwar eine Patientin wegen pyämischer Kniegelenksentzïndung, die beiden anderen, weil grössere, nicht ohrenärztliche operative Eingriffe erforderlich waren. Die Vertheilung der Kranken auf die einzelnen Jahrgänge ist aus folgender Tabelle ersichtlich:

\begin{tabular}{l|c|c|c|c}
\hline & Mănner & Weiber & Kinder & Summa \\
\hline \hline 1. April 1881 bis 1. April 1882 & 30 & 20 & 16 & 66 \\
1. April 1882 bis 1. April 1883 & 40 & 24 & 22 & 86 \\
1. April 1883 bis 1. April 1884 & 34 & 22 & 24 & 80
\end{tabular}

Die aufgenommenen 232 Kranken, von denen 113 an einseitiger, 119 an doppelseitiger Ohraffection litten, zeigten 240 verschiedene Krankheitsformen, deren Art und Vertheilung aus nachstehender Zusammenstellung hervorgeht: 


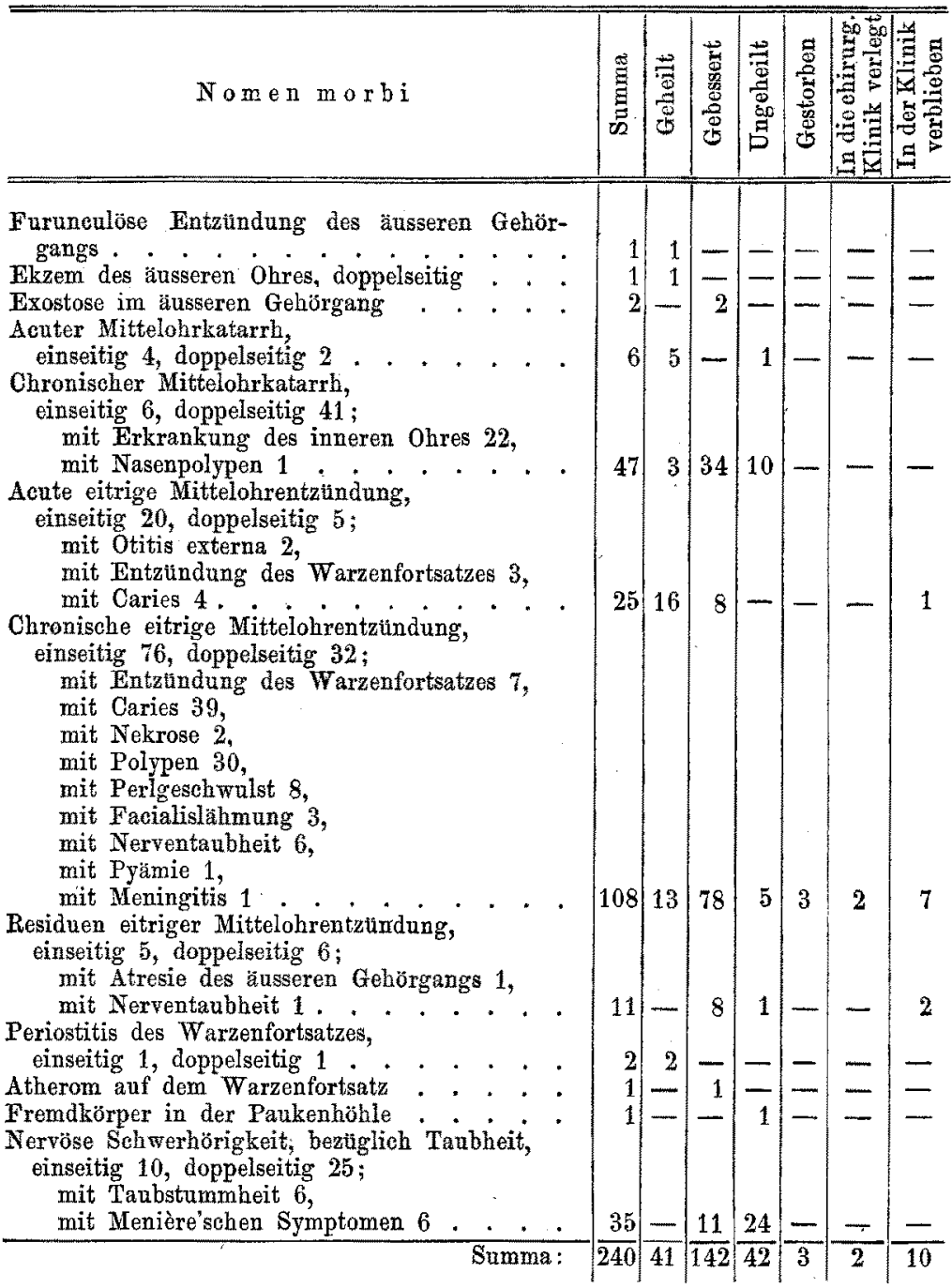

Die ausgeführten Operationen sind in der folgenden Uebersicht angegeben.

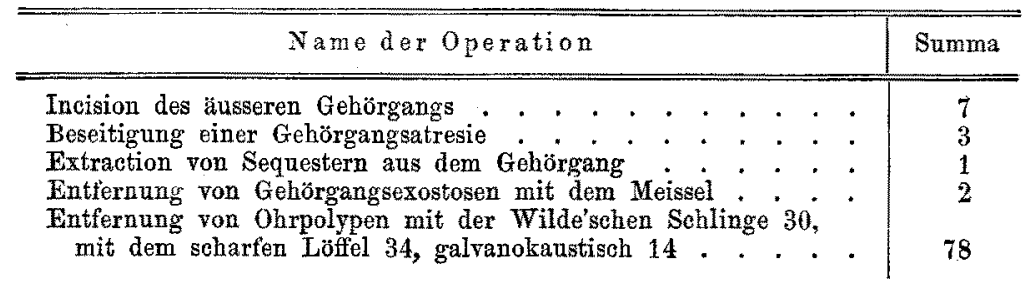




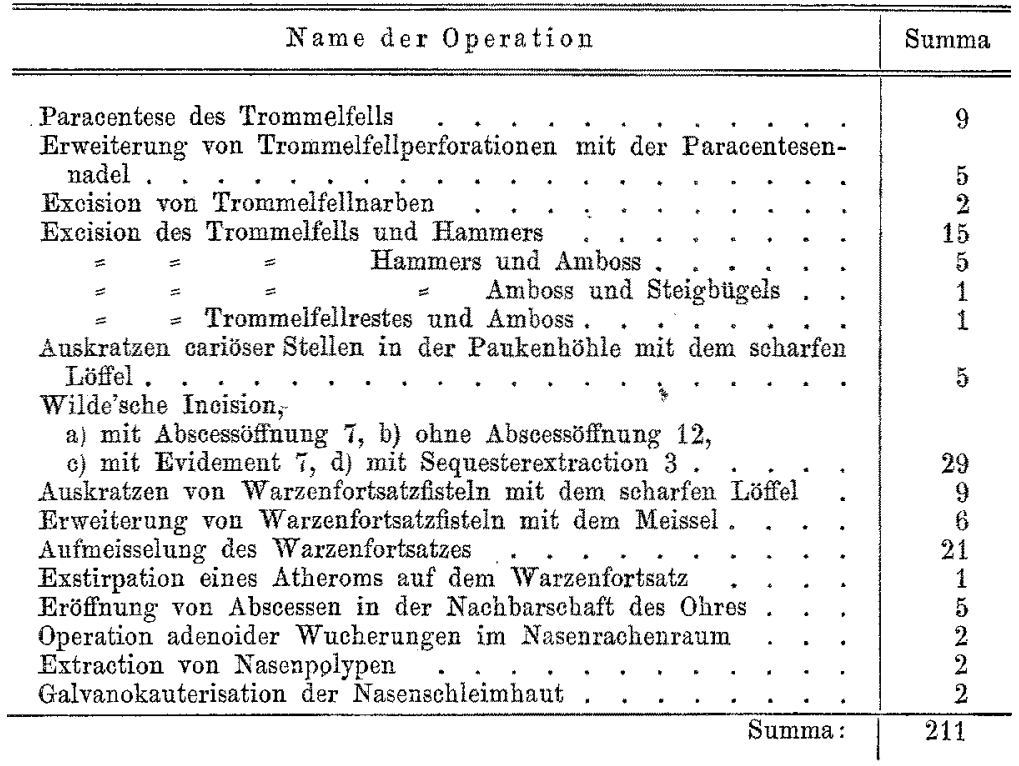

Zur Aufnahme in die Klinik wurden zunächst solche Kranke ausgewählt, bei denen grössere Operationen vorgenommen werden mussten, sodann solche, bei welchen die poliklinisch anwendbaren Behandlungsmethoden entweder von vornherein aussichtslos erschienen, oder sich im Laufe der Zeit als erfolglos erwiesen hatten, und bei welchen eine Sehwitzcur mittelst Pilocarpininjectionen eingeleitet werden sollte. In einer dritten Reihe von Fällen endlich. waren es äussere Umstände, welche die Aufnahme veranlassten. Hierhin gehören insbesondere Patienten mit schweren acuten Ohrentzindungen, bei welehen strenge Zimmerruhe wïnschenswerth erschien? nöthige Wartung and Behandlung zu Theil werden konnte.

Die Fälle, in welchen operative Eingriffe am Warzenfortsatz ausgefiihrt, und diejenigen, bei welchen wegen hochgradiger Veränderungen im schalleitenden Apparat Trommelfell and Gehörknöchelchen operativ entfernt wurden, wird Herr Prof. Lucae zum Gegenstand ausfuthrlicher Mittheilungen machen. Von den übrig bleibenden scheinen mir insbesondere diejenigen von Interesse, bei welchen subcutane Injectionen von Pilocarpin. hydrochlor. zur Anwendung kamen. In fast allen diesen Fällen, deren Gesammtzahl 35 beträgt, handelte es sich nach unseren heutigen Anschauungen um eine Erkrankung des schallempfindenden Apparates, welche $15 \mathrm{mal}$ mit einer Mittelohraffection 
combinirt war. Die Anwendung des Pilocarpins hatte den Zweck, durch energische Anregung der Schweiss- und Speichelsecretion etwaige, im inneren Ohr befindliche Exsudate oder nicht organisirte Entzündungsproducte zur Resorption zu bringen. Das Pilocarpin wurde subcutan applicirt und zwar bei Kräftigen 0,01, bei Schwächlichen 0,005 , bei Kindern 0,001-0,005 Grm., meist jeden dritten Tag, mitunter indessen auch seltener oder bei sehr robusten Patienten täglich. In der Regel begannen wir mit schwächeren Injectionen und gingen orst allmählich, wenn der Kranke dieselben gut vertrug, zu stärkeren ïber. Nicht selten indessen wurde im weiteren Verlaufe der Cur, falls Patient sich durch dieselbe sehr angegriffen fühlte, die Dosis wieder verringert. Dieser sehr vorsichtigen Anwendung des Mittels ist zuzuschreiben, dass in keinem der Fälle schwerere Intoxicationserscheinungen, wie Herzschwäche, Arythmie des Palses, Collaps, Dyspnoe eintraten. Geringere unangenehme Nebenwirkungen, wie Uebelkeit, Erbrechen, Diarrhoe, leichter Sehwindel gelangten indessen dennoch bei einigen Patienten zeitweise zur Beobachtung. Zur Untersiutzung der Wirkung wurden die Kranken unmittelbar nach der Injection in wollene Decken gehtillt, in denen sie in der Regel zwei Stunden lang blieben. Die Stärke der Schweissund Speichelsecretion schien individuell versehieden zu sein; auch hatte es den Anschein, als wenn nach öfterer Wiederholung der Injectionen die Secretion reichlicher erfolgt, als im Beginn der Cur. Was die therapeutischen Erfolge der letzteren anlangt, so hat Herr Prof. Luca in seiner Bearbeitung der Labyrinthkrankheiten ${ }^{1}$ ) dieselben bereits besprochen. Sein Urtheil lautet, dass in der Mehrzahl der Fälle, namentlich der ganz veralteten, nur Misserfolge, resp. unbedeutende, in praktischer Beziehung kaum in Betracht kommende Hörverbesserngen za verzeichnen sind, dass aber mitunter, wem auch selten, bedeutende bleibende Besserung erzielt wird, und dass man daher selbst bei chronischen Labyrinthaffectionen, anstatt alle Hoffnung aufzugeben, immer einen Versuch mit Pilocarpininjectionen machen solle.

Diese Auffassung von der Wirksamkeit des Pilocarpins wird auch durch die neueren, seitdem in der Klinik gewonnenen Erfahrungen an Kranken durchaus bestätigt. Ich füge hinzu, dass sie in gutem Einklang steht mit den diesbezüglichen Mittheilungen, welche Politzer bereits auf dem zweiten otologisehen Congress

1) Real-Encyclopädie der gesammten Heilkunde. Herausgegeben von Eulenburg. XV. Bd. ธ. 209. 
in Mailand im Jahre 1880 und später in seinem Lebrbuch der Ohrenheilkunde gemacht hat, und ferner mit neueren Beobachtungen von $\left.M \circ o^{1}\right)$ und 0 . Wolf $\left.{ }^{2}\right)$. Nar scheint es, als wenn diese Autoren die Pilocarpincur ausschliesslich in Fällen frischer Erkrankung wirksam fanden, während Lucae auch bei chronischen Labyrinthleiden einzelne, wenn auch sehr seltene Erfolge mit derselben erzielt hat. Es hätte sich das therapeutische Gesammtresultat dieser Behandlungsmethode vielleicht günstiger gestaltet, wenn nicht die überwiegende Mehrzahl der in die Klinik aufgenommenen hierher gehörigen Kranken mit den schwersten Formen veralteter Nerventaubheit behaftet gewesen wäre.

Die Zahl der von uns vorgenommenen Injectionen betrug in den Fällen, in welchen eine Besserung nicht eintrat, im Durchschnitt 10, wo dagegen Besserung constatirt werden konnte, wurde die Behandlung fortgesetzt, so dass bei einem Patienten sogar 44 Einspritzungen gemacht worden sind.

Ich erlaube mir, einige der interessanteren Fälle, namentlich die, in welchen ein günstiges Resultat sich ergab, obwohl die letzteren bereits von Herrn Prof. Lucae (a. a. O.) kurz angefuhrt wurden, an dieser Stelle etwas ausfiihrlicher mitzutheilen.

\section{Acute Erkrankung des schallempfindenden Apparates nach plötz- lichem Ausbleiben der Menses. Heilung durch Pllocarpin.}

Erna Eisenstedt, 18 Jabre alt, Verkäuferin aus Berlin. A ufnahme in die Poliklink am 7 . December 1881. Seit zwei Jahren besteht links permanentes Sausen und Schwerhörigkeit. In beiden Gehörgängen Ceruminalpfröpfe, welche ausgespritzt werden. Danach beiderseits Fl. $6(3)^{3}$ ), Sausen links unverändert. Trommelfelle normal.

1) Zeitschrift f. Ohrenheilk. XIII. Bd. S. 162.

2) Correspondenzblatt f. schweizer Aerzte. XIII. Jahrgang. 1883. S. 312.

3) Zux Erklärung der in den Krankengeschichten vorkommenden $\mathrm{Ab}-$ kürzungen bemerke ich Folgendes: Es bedeutet $\mathrm{R}$ sch. Fl. a. 0. (3) oder L Fl, 5, (Kakadu): Rechts wird das mit scharfer Flüstersprache gesprochene Wort "drei" nur dicht am Ohr verstanden, links beträgt die Hörweite für das mit Flüstersprache gesprochene Wort "Kakadu" 5,5 Meter, also das in der Parenthese befindliche Wort ist dasjenige, welches der Kranke bei der Hörprüfung dem Untersucher richtig nachspricht.

Rinne + bedentet: Der Rinne'sche Versuch fällt positiv aus, $d . h$. die $c$-Gabel wird, dicht vor die Ohroffnung gehalten, also per Luftleitung länger gehört, als beim Aufsetzen auf den Warzenfortsatz, also per Knochenleitung.

Rinne - bedeutet negativen Ausfall des Rinne'schen Versuches, also das Umgekehrte. 
Catheterismus links ohne Erfolg für das Sausen. Ordination: Kal. bromat. 1,0 zweimal täglich innerlich.

20. Januar 1882. Seit 8 Tagen auch rechts Sausen, das in beiden Ohren dem Waldesrauschen gleicht, zu welchem mitunter noch Klingen hinzukommt.

R Fl. 2 (3), c-50", fis ${ }^{4}-8^{\prime \prime}$, Rinne + , c vom Warzenfortsatz viel kürzer wie normal gehört.

L Fl. 4 (3), c-60", fis ${ }^{4}-4^{\prime \prime}$, Rinne +, c vom Warzenfortsatz viel kürzer wie normal gehört.

Contra-g, $c, c^{1}, c^{2}, c^{3}, c^{4}, f i s^{4}-x^{\prime \prime}$ bedentet: Die auf Contra-g, $c, c^{4}$ etc. abgestimmte Stimmgabel, beliebig stark angeschlagen, wird, dicht vor die Ohröffnung des Kranken gehalten, von letzterem beim Ausklingen um $\times$ Secunden weniger lange gehört, als von dem normal hörenden Ohre des Untersuchers. In letzterer Beziehung ist zu bemerken, dass wir aus den bei verschiedenen Kranken gefundenen Werthen von $x$ einen Schluss auf die relative Hörschärfe derselben nicht ziehen dürfen. Wenn also c bei einem Patienten $-20^{\prime \prime}$, bei dem anderen $-10^{\prime \prime}$ gehört wird, so bedeutet das nicht etwa, dass die Hörschärfe des ersteren für c $1 / 2$ mal so gross ist, als die des zweiten, und ebensowenig, dass sie in irgend einem anderen aus den gefundenen Werthen von $\mathrm{x}$ gesetzmässig abzuleitenden Verhältniss zu einander stehen. Denn nach Hensen (Physiologie des Gehörs im III. Bd. des Handbuchs der Physiologie, herausgegeben von Herman $\mathbf{n}, \mathrm{S} .120$ ) ist das logarithmische Decrement der Schwingungsamplitude beim Abklingen von Stimmgabeln keine Constante.

Noch weniger dürfen wir aus den verschiedenen Werthen von $x$, welche bei der Prüfung mit ungleich abgestimmten Gabeln sich ergeben haben, Schlüsse auf die relative Hörschärfe des betreffenden Ohres für verschieđen hohe Töne ziehen. Denn das Abklingen erfolgt bei Stimmgabeln von verschiedener Höhe in ganz differenter Weise; so braucht $z$. B. die von uns benutzte $\mathrm{c}$-Gabel, welche selbst bei stärkstem Anschlag einen so schwachen Ton gibt, dass sie $10 \mathrm{Cm}$. weit vom Ohr entfernt nicht mehr gehört wird, bis zum völligen Aus-

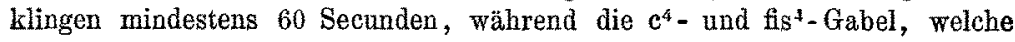
schon bei schwachem Anschlag noch 4--5 Meter weit deutlich gehört werden, nach stärkstem Anschlag höchstens 20-30 Secunden zum Ausklingen brauchen. Es hängt dieses nicht nur von der Höhe des Tones, auf den die Gabeln abgestimmt sind, sondern auch von der Form, der Grösse, dem Material derselben ab. Wenn demnach in den Krankengeschichten die Differenz der pathologischen Hörzeit von der normalen, also $x$, in Secunden ausgedrückt wird, so geschieht dieses nur, weil bei allen hier angeführten Kranken dieselben Stimmgabeln benutzt worden sind, und insofern unter Berücksichtigung der eben erörterten Schwierigkeiten Vergleiche sich anstellen lassen. Ich bemerke aber nochmals ausdrücklich, dass die Intensität der von den tiefen Gabeln (c und Contra - $g$ ) hervorgebrachten Töne selbst bei allerstärkstem Anschlag, wenn also bis zum Ausklingen etwa $60-70^{\prime \prime}$ vergehen würden, noch bei Weitem geringer ist, als die Intensität der von der $c^{4}$-oder fis ${ }^{4}$-Gabel hervorgebrachten Töne bei einer Differenz von nur $8-10^{\prime \prime}$. Dass wir bei Bestimmung vou $\mathrm{x}$ die vor das kranke Ohr des Patienten zu haltende Stimmgabel, um Uebertäubung zu vermeiden, in der Regel mit möglichst geringer Kraft anschlagen, bedarf kaum besonderer Erwähnung. 
Ordination: Sol. Kal, jodat. 4:200 3 mal täglich ein Esslöffel.

27. Januar. R sch. Fl. a. O. (3) bei geschlossenem und geöffnetem Gehörgang gleich gut, Contra - $g$ und c bei stärkstem Ansehlag nicht gehört, fis ${ }^{4}-20^{\prime \prime}$; Rinne -, e vom Warzenfortsatz 22" kürzer wie vom normalen.

L Fl. 7 (3, Friedrich).

Rechts ist das Sausen ein wirres Geräusch von sehr verschiedener Art, links wie das Heulen des Windes. Catheterismus rechts: Normales Auscultationsgeräusch, danach Fl. a. 0. (3).

Fiir ihr Ohrenleiden weiss Patientin keine Ursache, ausser dass seit 5 Wochen ihre frïher immer regelmässigen Menses ohne eine ihr bekannte Veranlassung ausgeblieben sind.

Aufnabme in die Klinik.

28. Januar. Ein Heurteloup auf den rechten Warzenfortsatz.

30. Januar. Sausen und Gehör unverändert.

Vom 30. Januar bis zum 9. März 1882 wurden nur subcutane Pilocarpininjectionen gemacht, im Ganzen 13, und zwar, da Patientin 0,005 Grm. nicht gut verträgt, à $0,0025 \mathrm{Grm}$. Bereits nach der fünften Injection R Fl. 0,8 (3) und Eintritt der Menses, welche zwei Tage dauerten; danach $\mathrm{R}$ Fl. 1,5 (3).

9. März. Entlassung aus der Klinik. Sausen beiderseits nur wenig vermindert, R Fl. 6 (3), 5, כ (5) 3 (Kakadu), c-48", fis ${ }^{4}-10$, Rinne-, c vom Warzenfortsatz $18^{\prime \prime}$ kïrzer wie vom normalen.

L Fl. 6,5 (Friedrich).

Zuletzt sah ich die Kranke am 19. October 1882. Das Gehör hatte sich unverändert gut erhalten. Das Sausen war, trotzdem Patientin inzwisehen von anderer Seite lange katheterisirt worden, trotzdem sie Amon. bromat. genommen und mit dem constanten Strom behandelt war, nicht geringer geworden.

Was die Diagnose des mitgetheilten Falles anlangt, so kann kaum zweifelhaft sein, dass es sich in demselben um eine Affection des schallempfindenden Apparates handelt, da das friber durchaus normal börende rechte $\mathrm{Ohr}$ in der kurzen Zeit von etwa 8-14 Tagen fast vollkommen taub warde, ohne dass die objective Untersuchung mit Spiegel und Katheter im schalleitenden Apparat Veränderungen nachzuweisen vermochte, welche eine derartige Functionsstörung hätten bedingen können.

Welchen Theil des schallempindenden Apparates die Erkrankung befallen hatte und welches die anatomische Natur derselben war, lässt sich mit Sicherheit nicht entscheiden. Da kurz vor dem Beginn des Ohrenleidens die sonst immer regelmässigen Menses ausgeblieben waren, so dürfte vielleicht an eine vicariirende Exsudation, resp. Blutung im Labyrinth gedacht werden.

Der in diesem Falle erzielte vorzingliche therapeutische Erfolg ist sicher dem Pilocarpin zu vindiciren. 


\section{Chronische Erkrankung des schallempfindenden Apparates. Bedeutende Besserung aurch Pilocarpin.}

Karl Leist, 32 Jahre alt, Kupferschmied ans Berlin. Aufnahme in die Klinik am 17. Januar 1882. Im Jahre 1876 kam Patient als Arbeiter in eine Torpedofabrik, woselbst er 2 Jahre hindurch Tag über in dem ausserordentlich heftigen Lärm, welchen eine grosse Zahl von Löthrohrstichfammen verursachte, beschäftigt war. Im Winter 1878 musste er oft auch die Nacht hindurch arbeiten. $\mathrm{Zu}$ dieser Zeit bemerkte er, dass das Geräusch der Stichflammen nach Verlassen der Werkstatt ihm Stunden lang im Kopf nachsummte. Zugleich verursachte ihm lautes anderweitiges Geräusch, wie das Hämmern in der benachbarten Kesselschmiede, Schmerz in den Obren. Im Frühjahr wurde er entlassen, behielt aber noch 4 Wochen lang sein mit dem von den Stichflammen verursachten Lärm identisches Sansen zurïek, nnd zwar hörte er dasselbe in den ersten 14 Tagen permanent, in den folgenden dagegen nur, sobald er daran dachte. Nach dem Aufhören des Sausens bemerkte Patient linkerseits Schwerhörigkeit.

Seit dem Sommer 1881 hört er zeitweise beiderseits pulsirendes "Picken" und bemerkt anch auf dem rechten Ohr eine Abnahme des Hörvermögens.

In den letzten Wochen beiderseits permanentes Zischen, links stärker als rechts.

Trommelfelle annähernd normal.

R Fl. 0,6 (3), c- $6^{\prime \prime}, \mathrm{e}^{4}-16^{\prime \prime}$, fis ${ }^{4}-8^{\prime \prime}$, Rinne + .

L Fl. 0,4 (3), $\mathrm{e}-18^{\prime \prime}, \mathrm{c}^{4}-17^{\prime \prime}$, fis $4-10^{\prime \prime}$, Rinne + .

Catheterismus beiderseits: Normales Auscultationsgeräusch, Gehör und Sausen danach unverändert.

Vom 17. Januar bis zum 9. Februar 1882 wurden 14 Pilocarpininjectionen a $0,01 \mathrm{Grm}$. gemacht.

9. Februar. Entlassung a us der Klinik. Sausen rechts beseitigt, links sehr gering.

R Fl. 4,5 (3), c-8", fis 4 -8", Rinne + .

L Fl. 0,9 (3), e-12", fis 4 -12", Rinne + .

In dem vorliegenden Fall stuitzt sich unsere Diagnose hauptsächlich auf das Ergebniss der Stimmgabeluntersuchung. Patient hörte das tiefe $c$ ausserordentlich viel besser, als das durchdringende hohe $e^{4}$ und fis ${ }^{4}$.

Besonderes Interesse scheint mir, abgesehen von dem therapeutischen Erfolg der Pilocarpincur, der von dem Patienten be-

1) Dieser Fall ist yon Herrn Prof. $L u$ cae in seiner nenerdings erschienenen Arbeit; „Zur Entstehung and Behandlung der subjectiven Gehörsempfindungen. Berlia 1884." bereits mitgetheilt worden. Da er indessen zu denjenigen gehört, bei welchen die in der Klinik eingeleitete Plocarpincur das herabgesetzte Hörvermögen ganz bedeutend besserte, so habe ich, um der Vollständigkeit meiner diesbezüglichen Mittheilungen willen es für angemessen gehalten, ihn hier noch einmal zu bringen. 
obachtete, wochenlang anhaltende Nachklang zu haben, welcher ihm durch das äusserst intensive schrille Geräusch der Löthrohrstichflammen verursacht worden war. In neuerer Zeit ist es namentlich Lucae (a. a. O.), welcher anf die Wichtigkeit dieser Nachempindungen hingewiesen hat.

Während im Fall 1 nur die Sehwerhörigkeit dareh die Pilocarpineur gebessert wurde, ist in dem vorliegenden auch das Sausen bedentend vermindert worden. Wir dürfen indessen nicht übersehen, dass der letztgenannte Erfolg zum Theil wenigstens dadurch erklärt werden kann, dass Patient während seines Aufenthaltes in der Klinik den fortwährenden acustischen Schädlichkeiten, denen er als Kupferschmied bisher sich aussetzen musste, entzogen war, ein für die Behandlung mancher subjectiven Geräusche äusserst wichtiges Moment, dessen Bedeutung Herr Prof. Lucae in der vorhin citirten Arbeit mit besonderem Nachdruck betont hat.

\section{Residuen eimer chronischen eitrigen Mittelohrentziindung. Besserung dureh Pilocarpin.}

Katharina Haake, 9 Jahre alt, Waise aus Berlin. A u $\mathrm{n}$ ah $\mathrm{me}$ in die Klinik am 30. März 1882. Seit 5 Jahren leidet das Kind zeitweise an linksseitiger Ohreiterung geringen Grades. Im März 1881 hat sie eine linksseitige Parotitis durchgemacht und ist seitdem angeblich anch rechts schwerhörig.

Das rechte Trommelfell stark verdickt, mässig eingezogen, links sehr grosse Trommelfellperforation.

R FI. 0,4 (3), fis ${ }^{4}-15^{\prime \prime}$, Rinne -.

L Fl. 0,5 (3), fis $4-12^{\prime \prime}$, Rinne -.

Patientin ist bereits etwa ein Jahr lang in der Poliklinik behandelt worden, und zwar erhielt sie, da das Gehör nach der Luftdouche links eher schlechter wurde und rechts unverändert blieb, längere Zeit hindurch kleine Gaben von Kal. jodat. innerlich, indessen gleichfalls ohne allen Erfolg.

In der Klinik wurden ihr subcutane Injectionen von Pilocarpin. hydrochlor. 0,0025-0,005 gemacht und zwar im Ganzen 19. Unter dieser Behandlung besserte sich das Hörvermögen des linken Ohres allmählich ziemlich bedentend, so dass bei der Entlassung am 7. Juni 1882 L Fl. 1,8 (3) gehört wurde. Rechts wurde eine Veränderung nicht erzielt.

Nach den ersten 8 Injectionen bemerkte ich links an der inneren Wand der Paukenhöhle mehrere stecknadelkopfgrosse Ekchymosen.

$O b$ in dem vorliegenden Fall linkerseits ausser dem schallleitenden Apparat, in welchem die Residuen der chronischen eitrigen Mittelohrentzündung objectiv nachweisbar waren, anch 
der schallempfindende erkrankt war, lässt sich mit Sicherheit nicht entscheiden. fis ${ }^{4}$ wurde links zwar ziemlich schlecht gehört, aber nieht in einem so hohen Grade, dass hieraus allein auf eine Affection des Nervenapparates zweifellos geschlossen werden dürfte.

$\mathrm{Zu}$ beriicksichtigen ist ferner, dass das Ergebniss der Stimmgabelprüfung hier nicht für ganz zuverlässig gelten darf, $\mathrm{da}$, wie das bei Kindern so häufig der Fall ist, die für eine solche Untersuchung nothwendige Aufmerksamkeit kaum erzielt werden konnte.

\section{Chronischer Mittelohrkatarrh. Besserung dureh Pilocarpin.}

Helene Buckpesch, 21 Jahre alt, Verkäuferin aus Berlin. Aufnahme in die Poliklinik am 20. April 1882. Seit einem Jahr besteht links Schwerhörigkeit und fast permanentes heftiges Ohrensausen.

L Fl. 1 (3), Trommelfell normal, bei Catheterismus links dünnes Blasegeräusch, danach Sausen vorüber, Gehör unverändert.

Da wiederholte Application des Katheters und innerliehe Darreichung von Kal. bromat. weder die Schwerhörigkeit, noch das Sausen erheblich besserten, wurden am 27. Juli 1882 mit einer einfachen Sonde 1) Stempelbewegungen am linken kurzen Hammerfortsatz vorgenommen; danach

L Fl. 2,5 (3), Sausen etwas geringer.

Am 16. März 1883 klagte Patientin, dass sie nunmehr auch rechts schwer höre.

R Fl. 2,5 (3), L Fl. 2 (3), Catheterismus beiderseits normales Auscultationsgeräusch, danach

R Fl. 3 (3), L Fl. 4 (3).

Am 9. April 1883 liess sich Patientin, da die durch den Catheterismus erzielte geringfügige Besserung des Hörvermögens immer nur kurze Zeit anhielt und auch trotz hänfiger Wiederholung nicht constant erhalten werden konnte, zur Einleitung einer Pilocarpincur in die Klinik aufnehmen.

Hier ergab die Untersuchung normalen Trommelfellbefund, beiderseits Fl. 2,5 (3), c vom Warzenfortsatz ebenso lange gehört, wie vom normalen Warzenfortsatz.

R $\mathrm{c}-70^{\prime \prime}, \mathrm{e}^{4}-9^{\prime \prime}$, fis $^{4}-8^{\prime \prime}$, Rinne -

L c $-55^{\prime \prime}, \mathrm{c}^{1}-10^{\prime \prime}$, fis ${ }^{4}-8^{\prime \prime}$, Rinne -

Beim Catheterismus normales Blasegeräuseh, danach

R FI. 2 (3), L FI. 2,5 (3) und Sausen links etwas geringer.

Patientin erhielt nun subcutane Injectionen von Pilocarpin. hydrochl. 0,005-0,01 Grm. und zwar bis za ihrer Entlassung am 19. Mai 1883 im Ganzen 19.

1) Die von Herrn Prof. Lucae (s. S. 84 dieses Bandes) beschriebene und jetzt zu den von ihm empfohlenen "Stempelbewegungen" allein benutzte „federnde Drucksonde" war damals noch nicht construirt.

Arehiv f, ohrenhoilknde. XXI. Ba. 
Unter dieser Behandlung besserte sich das rechte $\mathrm{Ohr}$ bedeutend, so dass bei der Entlassung R Fl. 7,5 (3, 8,1) gehört wurde.

Eine Veränderung des Sausens sowie der linksseitigen Schwerhörigkeit trat nicht ein.

Wenn in dem vorletzten Falle bereits zweifelhaft bleiben musste, ob der schallempfindende Apparat gesund oder erkrankt war, so ist dieses in dem zuletzt mitgetheilten in noch höberem Maasse der Fall.

Die Stimmgabelprüfung ergab eine anscheinend gleichmässige Herabsetzung der Hörschärfe für hohe und tiefe Töne $\left(c, e^{4}\right.$ fis $\left.^{4}\right)$, der Rinne'sche Versuch fiel negativ aus. Hieraus also würde man auf eine Erkrankung des schallleitenden Apparates schliessen, für welche auch das beim Catheterismus zeitweilig beobachtete dünne Auscultationsgeräuch spräche. Indessen kann weder das letztere, noch der Ausfall der Stimmgabeluntersuchung daza dienen, eine gleichzeitige Affection des inneren Ohres auszuschliessen.

Will man eine solche nicht acceptiren, so bleibt nichts übrig, als die nicht unbedeutende Steigerung der Hörschärfe auf eine der Pilocarpincur zu verdankende Besserung des chronischen Mittelohrkatarrhs zu beziehen, eine Annahme, welche nach meiner Ansicht gleichfalls durchaus nichts Unwahrscheinliches enthält.

\section{Nerventaubheit nach Meningitis. Besserung dureh Pilocarpin.}

Frau Weisser, 39 Jahre alt, aus Berlin, fruher vollkommen gesund, erkrankte Mitte Juli 1882 plötzlich mit starkem Erbrechen, Stuhlverhaltung, äusserst intensivem Kopfschmerz und hohem Fieber. Der Kopf, vom ersten Tage an stark nach hinten gezogen, konnte nicht nach vorn gebeugt werden. Das Bewusstsein blieb bis auf zeitweise auftretende Delirien ungetrübt. Am 3. Tage der etwa 8 Wochen danernden Erkrankung trat Schwerhörigkeit ein, die sich bis zum 9. Tage zu so hochgradiger Taubheit steigerte, dass laute Sprache am Ohr nicht mehr gehört wurde. Zugleich mit der $\mathrm{Ab}$ nahme des Hörvermögens stellte sich auch Summen im Kopf und Schwindel ein, anfangs heftig, allmählich indessen an Intensität abnehmend.

Am 2. December 1882 liess sich Patientin, die bis auf mässigen, beim Gehen eintretenden Schwindel, Ohrensausen und Schwerhörigkeit wieder vollkommen hergestellt worden war, in die Ohrenklinik aufnehmen.

Hier fanden wir das rechte Trommelfell stark eingezogen, das linke annähernd normal. Beiderseits mässig laute Sprache a. 0 . (3). R c $-45^{\prime \prime}, \mathrm{c}^{4}-14^{\prime \prime}, \mathrm{fis}^{4}-17^{\prime \prime}$, Rinne + .

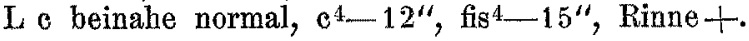


Bei Catheterismus normales Auscultationsgeräusch, danach Gehör nnd Sausen unverändert.

Patientin blieb in der Klinik bis zum 8. Januar 1883 und erhielt im Ganzen 15 subeutane Pilocarpininjectionen von je 0,0075 Grm. Unter dieser Behandlung nahmen Ohrensausen und Schwindel bedeutend $a b$, und auch das Gehör besserte sich etwas. Bei der Entlassung hörte sie R sch. Fl. a. O. (3) und L Fl. 0,3 (3).

Für die Stimmgabeltöne wurde eine Besserung nicht erzielt.

\section{Doppelseitige Menière'sche Erkrankung. Bedentende Besserung durch Pilocarpin.}

Albrecht Reimer, 32.Jahre alt, Arbeiter aus Berlin, fruiher vollkommen gesund, erwachte am 22. Januar 1883 mit sehr heftigem, gleichmässigem Zischen im Kopf and in den Ohren. Gleichzeitig bemerkte er starke Schwerhörigkeit beiderseits, namentlich aber rechts, and permanentes Sehwindelgefühl, selbst im Liegen. Es schien ihm, als wenn sich die Gegenstände im Zimmer von einer Seite zur anderen zuckend hin-und herbewegten. Allein konnte Patient gar nicht gehen, weil er dabei von einer Seite zur anderen taumelte. Bei längerem Stehen fiel er um, angeblich nach rechts und hinten. Ausserdem bestanden fortwährende klopfende Schmerzen im Kopfe, namentlich heftig in der Stirn, und in den ersten 8 Tagen etwa 2 mal täglich Erbrechen bei nüchternem Magen.

Früher hat Patient seiner Angabe nach nie an den Ohren gelitten und immer vollkommen gut gehört.

Am 25. Januar 1883 suchte er die Poliklinik auf. Inzwischen soll sich das Gehör linkerseits von selbst etwas gebessert haben.

Die Cntersuchung ergab L Fl. 1 (3), R vollkommene Taubheit für Sprache. Trommelfelle ein wenig getrübt, Gehörgänge im inneren Theil etwas geröthet; bei Catheterismus beiderseits normales Auscultationsgeräusch, danach keine Veränderung. Mit Ausnahme der Acustici keine Lähmungserseheinungen im Bereiche der Hirn- und Rückenmarksmerven. Ordination: Sol. Kal. jodat. $6: 300$ dreimal täglich ein Esslöffel.

27. Januar. Schwerhörigkeit und subjeetive Beschwerden haben zugenommen.

29. Januar 1883. Aufnahme in die Klinik.

Stat. praes. Ohrenspiegelbefund wie am 25. Januar 1883.

$\mathrm{R}$ Taubheit für Sprache, c, $\mathrm{e}^{4}$ und fis ${ }^{4}$ bei stärkstem Anschlag nicht gehört, vom Warzenfortsatz wird e gehört, aber etwas kürzere Zeit, wie normal. Rinne-.

L Fl. 0,4 (3), c-25", $\mathrm{e}^{4}-14^{\prime \prime}$, fis 4 -14", Rinne +.

c per Kopfknochenleitung von einigen Punkten der Mittellinie nach rechts, von anderen nach links, von der rechten Kopfhälfte nach rechts, von der linken nach links gehört.

Subjective Beschwerden wie am 25. Januar 1883. Zunge sehr belegt, Puls 64 .

30. Januar. Seit 2 Tagen keine Stuhlentleerung. Ordination: 
Auf jeden Warzenfortsatz zwei Heurteloups, innerlich Calomel, Pulv. rad. Jalapp. ana 0,25 .

31. Jannar. Stat. idem. Ordination: Acid. hydrobromic. dreimal täglich 15 Tropfen einzunehmen.

2. Februar. Stat. idem. Seit 24 Stunden kein Stuhl. Ordinatio n: Ol. Ricini.

5. Februar. Kopfschmerz vorüber, Schwindel etwas geringer, 80 Pulse, sonst Stat. idem.

Vom 5.-12. Februar täglich O1. Ricini wegen anhaltender $\mathrm{Ob}$ stipation. Hörvermögen unverändert.

Vom 12. Februar an wurden Pilocarpininjectionen (a $0,01 \mathrm{Grm}$.) angewandt und zwar bis zum 24. April im Ganzen 32. Nebenbei wurde abwechselnd Acid. hydrobrom. und Sol. Kal. jodat. verabreicht und wegen hartnäckiger Obstipation durch Abfithrmittel für regelmässige Stuhlentleerung gesorgt.

Während dieser Behandlung nahm der Schwindel bedeutend ab und zwar bereits nach wenigen (5) Injectionen, kehrte aber im weiteren Verlauf zeitweise, wenn anch in schwächerem Grade wieder.

Am 18. März nach 13 Pilocarpininjectionen L FI. 1 (3).

21. März. L Fl. 1,5 (3).

24. April. Nachđem das Gehör inzwischen wegen eines intercurrenten Schnupfens starke Schwankungen gezeigt hatte, und im Ganzen 32 Injectionen gemacht waren, L Fl. $8(3,1,8) 5(20,5)$, c, $\mathrm{e}^{2}$, $\mathrm{e}^{4}$, fis ${ }^{4}-0^{\prime 4}$, $\mathrm{e}^{3}$ nicht ganz bis zu Ende, o per Knoehenleitung von der Mittellinie und der linken Hälfte des Kopfes nach links, von der rechten Kopfhälfte nach rechts gehört.

Beim Gehen mit geschlossenen Augen fallt Patient nach rechts hinüiber.

14 Tage werden die Pilocarpininjectionen ausgesetzt und statt dessen stärker laxirt. Danach angeblich das Sausen geringer.

30. April. Seit heute geringe Schmerzen auf dem rechten Warzenfortsatz spontan und bei Druck. Bei jedem lauten Geräusch fühlt Patient nach seiner Angabe im rechten Ohre starkes Zucken. Heurteloup auf den rechten Warzenfortsatz, danach Schmerz vorüber.

1. Mai. Patient hat beobachtet, dass das bei lautem Schall im rechten Ohre auftretende Zucken bei. Versehluss des linken Ohres ausbleibt. Dieses ergibt auch die Untersuchung am Harmonium.

9. Mai. Patient fühlt beim Anschlagen von Stimmgabeln vor dem linken Ohre kurzes Zucken im rechten Ohre, das aber nur so lange dauert, wie der Anschlag. Beim Ausklingen der Gabeln vor dem linken Ohre fühlt er rechts kein Zucken, wohl aber eine $\mathrm{Zu}$ nahme seines Sausens.

Vom 9. Mai ab klagt der Kranke wieder über stärkeres, an Intensität sehr wechselndes Sausen und zeitweise auftretende Schwerhörigkeit links. Infolge dessen werden wiederum Pilocarpininjectionen (à $0,01 \mathrm{Grm}$.) gemacht und zwar bis zum 23. Juni im Ganzen noch 12. Das Sausen wird hierdurch nieht beseitigt und werden 
deshalb am 25. Juni auf beide Warzenfortsätze Vesicantien applicirt und mit Reizsalbe verbunden. Momentan war das Sausen hiernach geringer, bald aber nahm es wieder zu.

3. Juli 1883. Entlassung des Patienten.

Stat. praes.: Sausen noch immer stark, indessen an Intensität sehr wechselnd. Schwindel seit 3 Wochen vollkommen verschwunden.

R a. O. mässig laute Sprache, c bei stärkstem Anschlag nicht, vom rechten Warzenfortsatz nach links gehört, $\mathbf{c}^{4-24^{\prime \prime}}$. - L Fl. 9 (Zwieback, Friedrich, Marcipan, 3, 1, 8, 6, 20,4), c-15", vom linken Warzenfortsatz $12^{\prime \prime}$ kürzer wie in der Norm, $\mathrm{c}^{4}$ und fis ${ }^{4}-0^{\prime \prime}$. Rinne + .

Da die objective Untersuchung des Patienten mittelst Ohrenspiegel und Katheter wenige Tage nach Beginn seiner Erkrankung vorgenommen wurde, ohne dass wesentliche anatomische Veränderungen sich nachweisen liessen, so ist der Sitz des Leidens für das rechte $\mathrm{Ohr}$, auf welchem für Sprache völlige Taubheit bestand, unseren heutigen Anschauungen gemäss mit Sicherheit jenseits des Mittelohres zu suchen. Aber auch für das linke scheint mir eine andere Annahme nicht statthaft zu sein, vorausgesetzt, dass wir der Angabe des Kranken, nach welcher er bis zum 22. Januar 1883 beiderseits vollkommen normal gehört hätte, Glauben schenken wollen. Denn eine rasch entstandene, so hochgradige Schwerhörigkeit, wie die des linken Ohres, hätte als Ursache viel erheblichere objectiv nachweisbare Veränderungen im schallleitenden Apparat erfordert, als die hier vorhandenen, und hiermit steht in Uebereinstimmung das Ergebniss der Stimmgabelprüfung, insofern die hohen Töne $\mathrm{c}^{4}$ und fis ${ }^{4}$ ungleich schlechter percipirt wurden, als das tiefe c. Da das Leiden ganz plötzlich ohne Vorläufer unter Menière'schen Symptomen eintrat, da ausser im Bereiche des Hörnerven Lähmungserseheinungen nicht nachzuweisen waren, und dem vorher Gesagten zufolge eine Affection des schallleitenden Apparates als causales Moment ausgeschlossen werden darf, so konnte es sich hier nur um doppelseitige Menière'sche Erkrankung handeln. Politzer sagt bei der Besprechung dieser Affection in seinem Lehrbuch, das Hörvermögen sei hier entweder vollständig erloschen oder wenigstens so sehr herabgesetzt, dass nur noeh sehr starker Schall in unmittelbarer Nähe des Ohres wahrgenommen werde. Dieser Angabe wïrde unser Patient widersprechen, da er bereits 3 Tage nach dem Anfall links Fliustersprache 1 Meter weit vom $\mathrm{Ohr}$ entfernt hörte. Dennoch glaubten wir, bei der im Uebrigen charakteristischen Eigenthümlichkeit des mitgetheilten Krankheitsbildes eine andere Diagnose nicht stellen zu können. 
Das Hauptinteresse in dem vorliegenden Fall beansprucht der therapeutische Erfolg, insofern eine vollkommene Wiederherștellung des Hörvermögens, wie sie hier linkerseits erzielt wurde, nicht häufig beobachtet sein dürfte. Welchen Antheil an diesem erfreulichen Resultat die Pilocarpininjectionen haben, lässt sich mit Sicherheit nicht feststellen, da neben ihnen noch Heurteloups, Kal. jodat., Acid. hydrobromic. und Laxantien angewandt worden sind. Mit Rüeksicht auf die vorher mitgetheilten Erfahrungen ist unter diesen Mitteln doch wohl dem Pilocarpin die entscheidende Wirkung beizumessen, und den ibrigen nur eine unterstïtzende Kraft zuzugestehen.

Von nicht geringem physiologischen. Interesse scheinen mir zwei Erscheinungen $\mathrm{zu}$ sein, welche Patient selbst an sich beobachtet hat, zunächst das im Beginn der Erkrankung bemerkte ruckweise sich Hin- und Herbewegen der Gesichtsobjecte, eine Empfindung, die wohl nur durch nystagmusartige Bewegungen der Bulbi erklärt werden kann.

Noch mehr der Beachtung werth indessen erscheint mir jenes kurze Zucken, welches Patient vom 30. April ab in seinem rechten tauben Ohre spürte jedesmal in dem Moment, wo dem linken bereits ziemlich feinhörenden ein lauter Schall zugeführt wurde. Wiewohl bei diesem Zucken eine Bewegung am rechten Trommelfell nicht constatirt worden ist, so kann dasselbe nach meiner Ansicht doch nur durch eine Contraction der Binnenmuskeln des rechten Ohres, entweder des Tensor tympani, oder des Stapedius, oder vielleicht beider zusammen erklärt werden. Wir hätten diese Muskelznckung als eine reflectorisch durch Reizung des linken Hörnerven bedingte zu betrachten, ähnlich den von Hensen ${ }^{1}$ ) und später von Bockendahl ${ }^{2}$ ) an Thieren experimentell erwiesenen Zuckungen des Tensor, resp. Stapedius am Anfang jeder Schallerregung.

Ausser dem eben mitgetheilten sind in der Klinil noch 4 andere Fälle von Menière'scher Erkrankung behandelt worden. Allerdings konnte in diesen nur eine Wahrseheinlichkeitsdiagnose gestellt werden, da sie erst längere Zeit nach Beginn des Leidens zur Untersuchung gelangten, und somit eine acute Trommelhöhlenaffection als Ursache der unter Schwindel, Sausen und Brechneigung plötzlich aufgetretenen hochgradigen Schwerhörigkeit nicht ausgeschlossen werden konnte.

1) Arch. f. Physiologie. 1878. S, 312.

2) Dieses Archiv. XVI. Bd. S. 241. 
Auch bei diesen vier Kranken wurde die Pilocarpincur angewandt, indessen hier leider ohne Erfolg für das Hörvermögen. Einmal standen wir von weiteren Injectionen $a b$, da die betreffende Patientin gleich nach der ersten mit voller Bestimmtheit angab, ihr Sausen sei dureh das Sehwitzen viel stärker geworden, und höre sie seitdem auch mit dem bisher gesunden Ohre entschieden schlechter, Erscheinungen, welche allerdings nach circa 8 Tagen wieder verschwanden.

Der folgende Fall gehört zu den relativ seltenen, in welchen das Ohrenleiden mit einem apoplectiformen A fall einsetzt.

\section{Doppelseitige Menière'sche Erkrankung.}

Johann Dimke, 39 Jahre alt, Briefträger aus Kleinitz, verlor am 8. Mai 1883, nachdem er bis dahin vollkommen gesund gewesen war, beim Herabsteigen von einer Leiter ohne die geringsten Vorboten plötzlich das Bewusstsein nnd fiel die beiden letzten Sprossen herunter. Die Bewusstlosigkeit soll etwa zwei Stunden gedauert haben. Dann fand man ihn und bemerkte sogleich vollkommene Taubheit. Er antwortete auf geschriebene Fragen mit ungewöhnlich lauter Stimme, aber vollkommen zusammenhängend und verständig. Nach dem Anfall bat Patient mehrmals erbrochen und beim Gehen so stark getaumelt, dass er von zwei Männern geführt werden musste. Die ersten 4 Wochen war der Schwindel ausserordentlich heftig und musste Patient deshalb im Bette bleiben. Unmittelbar nach dem Anfall sollen nach Angabe des Patienten subjective Gehörsempfindungen nicht bestanden haben, und erst nach melreren Tagen heftiges Sausen eingetreten sein. Die Mutter indessen erzählt, er habe bereits in den ersten Tagen häufig gerufen: „bringt mir doch den Wind weg “. Lähmungserseheinungen sind ausser im Bereiche des Hörmerven nicht beobachtet worden.

A ufahme in die Klinik am 22. September 1883.

Laute Sprache a. 0 . wird beiderseits gehört, aber nicht verstanden.

R c, $\mathrm{e}^{4}$, fis ${ }^{4}$ bei stärkstem Anschlag nicht gehört, vom rechten Warzenfortsatz dagegen wird c gehört, also Rinne -.

L c-85", $\mathrm{c}^{4}$ nur bei stärkstem Anschlag, fis ${ }^{4}$ bei stärkstem Anschlag nicht gehört, Rinne -.

Rechts starkes, links geringes Sausen. Trammelfelle normal.

7. November 1883. Patient wird, nachdem inzwischen 26 Pilocarpininjectionen (a $0,01 \mathrm{Grm}$.) gemacht worden sind, ungebessert entlassen.

In dem vorliegenden Falle könnte man daran denken, die unter Menière'schen Symptomen aufgetretene Taubheit auf eine traumatische Affection des Ohrlabyrinths zu beziehen. Da der Kranke indessen nur die untersten beiden Sprossen der Leiter verfeblte, also aus ganz geringer Höhe herunterfiel, so balte ich 
es für richtiger, die apoplektische Form des Morbus Menieri hier anzunehmen, welche durch Zufall das Herabfallen von der Leiter herbeifübrte.

Was die beiden noch nicht mitgetheilten Fälle anlangt, in denen wir die Diagnose auf einseitige Menière'sche Krankheit stellten, so möchte ich auf eine ausfiuhrliche Wiedergabe der betreffenden Krankengeschichten verzichten, erlaube mir aber aus denselben einige Angaben bervorzuheben, welche theils in physiologischer, theils in klinischer Hinsicht Interesse beanspruchen.

Zunächst hätte ich gewisse Sehstörungen anzuführen, ähnlich denjenigen, welche in Fall 6 erwähnt wurden.

Eine Patientin (Fall 8) sah in den ersten 3 Tagen nach der Erkrankung alle Gegenstände doppelt, und schienen ihr dieselben sich zuckend hin- und herzubewegen, während sie später, obwohl nach dem ersten Anfall noch 4 Wochen hindurch heftiger Schwindel bestand, weder Doppeltsehen, noch eine Scheinbewegung der Gesichtsobjecte bemerkt hat.

Einer zweiten Patientin (Fall 9) schienen die Gegenstände während der ersten Schwindelattaque gr ü $\mathrm{n}$ auszusehen und sich von links nach rechts (das rechte Ohr war das kranke) im Kreise zu drehen; auch bestand dabei Photopsie. Diese Kranke hatte ausser permanentem, bei Bewegungen zunehmendem Schwindelgefithl alle paar Tage starke Schwindelanfälle, und bei jedem derselben schienen ihr die Gesichtsobjecte wiederum sich von links nach rechts im Kreise zu drehen; mitunter sah sie dabei auch Funken. Während der Anfülle wurde ihr nach Angabe der Angehörigen der Kopf ganz roth.

Eine fernere Beobachtung, die ich nicht unerwäbnt lassen möchte, bezieht sich auf die subjectiven Geräusche der beiden letzten Patienten. Dieselben hatten seit Beginn ihres Ohrenleidens kllopfendes, mit dem Radialpuls isochrones Sausen. Letztere Erscheinung steht in einem gewissen Widerspruch zu den Angaben Menière's, welcher ausdrücklich hervorhebt, dass nach seinen bei einigen Kranken angestellten Erhebungen die Geräusche niemals einen pulsirenden Charakter zeigten, woraus sich ihre vom Blutstrom unabhängige Natur ergäbe. 1) Wiewohl

1) Der betreffende Passus der Menière'schen Arbeit „Mémoire sur des lésions de l'oreille interne donnant lieu à des symptomes de congestion cérébrale apoplectiforme" in der Gazette médicale de Paris 1861. No. 38 p. 597 lautet: „Chez quelques malades plus attentifs à ce qui se passe en eux, il me fut possible à l'aide de questions très-précises, d'établir que les vertiges, 
diese Bemerkung sich nur auf einige Kranke bezieht, so hat es den Anschein, als wenn Menière in dem nicht pulsirenden Charakter des Ohrensausens bei der von ihm beschriebenen Affection des Gehörorgans ein pathognostisehes Merkmal findet, und insofern widersprechen unsere oben angeführten Beobachtungen den seinigen. Trotzdem glaube ich, auch in diesen beiden Fällen die Diagnose nicht anders stellen zu können, da die betreffenden Kranken im Uebrigen dentlich den charakteristischen Menière'schen Symptomencomplex zeigtén. Aus der Natur der subjeetiven Gehörsempfindungen einen Schluss auf den Sitz der zu Grunde liegenden Erkrankung zu ziehen, ist eben nicht statthaft, wie das anch Lucae in seiner diesen Gegenstand behandelnden Arbeit ${ }^{1}$ ) sagt.

Was die eigenthümlichen Sehstörungen anlangt, von denen ich vorhin gesprochen habe, so gestehe ich, fur das in Fall 9 im Beginn der Erkrankung beobachtete "Grïnsehen " eine genügende Erklärung nicht geben zu können. Die Photopsie, welche die betreffende Patientin wärend ihrer Schwindelanfälle bemerkte, möchte ich mit Rücksicht auf die Röthung des Kopfs, welche dabei regelmässig eingetreten sein soll, auf eine Reizung des Sehnervenapparats durch vorübergehende Hyperämie beziehen. Das scheinbare Hin- und Herzucken der Gegenstände, welches in Fall 6 und 8 angegeben wurde, weist mit Sicherheit auf Nystagmus der Augäpfel hin. $O b$ auch die in Fall 9) beobachtete scheinbare Drebung der Gesichtsobjecte im Kreise durch derartige Bewegung der Bulbi hervorgerufen sein kann, will ich dahingestellt sein lašsen, bekenne aber, dass für mich diese Annahme grosse Wahrscheinlichkeit zu haben seheint.

Suchen wir nun für das Zustandekommen der unwillkürlichen Augenbewegungen in den mitgetheilten Fällen eine Erklärung zu finden, so müssen wir uns zunächst daran erinnern,

l'état syncopal, la chute subite du corps, les vomissements avaient été précédés de bruits dans les oreilles, que ces bruits ne reconnaissaient aucune cause appréciable, qu'ils persistaient dans l'intervalle des accès, mais qu'ils coincidaient souvent avec l'angmentation des étourdissements, et $q u$ e $\mathbf{j a}$ mais ces bruits ne prenaient la formesaccadée, artérielle, en un mot qu'ils n'étaient pas carotidiens. C'était déjà un indice de leur charactère nerveux, ils dépendaient d' un état particulier des nerfs acoustiques et non d'une cause sanguine; le système circulatoire n'y était pour rien."

1) ,Zur Entstehung und Behandlung der subjectiven Gehörsempfindungen. Berlin 1884." S. 15. 
dass nach Angabe zahlreicher Autoren Nystagmus auftritt bei Reizung resp. Lähmung der Vestibularzweige des Hörnerven. In neuerer Zeit ist es namentlich $\mathrm{Högyes,} \mathrm{welcher} \mathrm{diesen} \mathrm{Gegen-}$ stand eingehend studirt hat. Nach seinen Untersuchungen treten bei Reizung des $\mathrm{N}$. acusticus sowohl, wie des häntigen Ohrlabyrinths verschieden charakterisirte bilaterale Augenbewegungen auf, deren Art von der Intensität und dem Angriffspunkt des Reizes abhängt. Und dasselbe ist der Fall bei Lähmung resp. Zerstörung der genamnten Theile.

Demnach wiürden die in unseren Fällen beobachteten Sehstörungen und der hieraus nothwendig resultirende Schwindel leicht erklärt werden können durch eine Affection des inneren Obres.

Bekanntlich ist es Menière selbst, welcher in einer solchen die Ursache der von ihm beschriebenen Krankheit findet. Neuere Forscher haben gegen diese Anschauung Widerspruch erhoben. Baginsky namentlich behauptet mit aller Sicherheit, dass eines der wichtigsten Symptome des Morbus Menieri, der Schwindel, durch eine Erkrankung des Ohrlabyrinths niemals ausgelöst werden könne and immer als Ausdruck einer Hirnläsion betrachtet werden müsse.

Obwohl diese Annahme Baginsky's bereits ron zahlreichen Autoren, wie Moos, O. Wolf, Spamer, Högyes, Bechterew, Landois u. A. als unhaltbar zurüekgewiesen ist, so scheint es mir dennoch nicht überflüssig, einen Fall mitzutheilen, welcher vielleicht geeignet sein dürfte, auf die in Rede stehende schwierige Streitfrage einiges Licht zu werfen:

\section{Schwindelanfalle bei Einwirkung des $e^{4}$.}

Adolph Sarnow, 36 Jahre alt, Kaufmann aus Bromberg. A ufnahme in die Klinik am 6. Juni 1882. Seit 5 Jahren besteht permanentes Sausen in beiden Ohren und eine zunehmende Schwerhörigkeit, die allmählich sehr hochgradig geworden ist.

Das rechte Trommelfell im unteren vorderen Quadranten stark verdickt, das linke etwas eingezogen und am Rande getrubt.

R. mässig laut a. O. (3) L laut a. O. (3).

$R$ Contrarg-60", c-5 $8^{\prime \prime}, \mathrm{c}^{3}-24^{\prime \prime}, \mathrm{c}^{4}-14^{\prime \prime}$, fis $\mathrm{s}^{4}-15^{\prime \prime}$ bei starkem Anschlag -18", c per Knochenleitung vom rechten Warzenfortsatz aus $-8^{\prime \prime}$ (mit dem normalen Warzenfortsatz verglichen), Rinne -.

L Contra-g - $45^{\prime \prime}, \mathrm{c}-49^{\prime \prime}, \mathrm{c}^{3}-17^{\prime \prime}, \mathrm{c}^{4}-9^{\prime \prime}$, bei starkem Anschlag - 12", fis ${ }^{4}-12^{\prime \prime}$, bei starkem Anschlag - 24", c per Knochenleitung vom linken Warzenfortsatz aus $-10^{\prime \prime}$ (mit dem normalen Warzenfortsatz verglichen), Rinne + . 
Hält man die starkangeschlagene $\mathrm{c}^{4}$-Gabel vor das rechteOhr des Patienten, so wird demselben schwindlig; es seheint ihm, als wenn sich die Gegenstände im Zimmer von links nach rechts um ihn herumdrehen; er selbst glaubt dabei nach vorn zu fallen. Die Gleichgewichtsstörung ist so stark, dass sich der Kranke, wenn er bei Anstellung des Versuchs auf eïnem Stuhl sitzt, unwillkürlich an demselben festzuhalten versucht.

Bei Catheterismus normales Auscultationsgeräusch. Da Patient bereits vielfach mit Luftdonche behandelt worden war, ohne dass eine nennenswerthe Besserung seines Leidens eingetreten wäre, so entfernte ihm Herr Prof. Lucae am 7. Juni 1882 in der Chloroformnarkose linkerseits Trommelfell, Hammer und Amboss, wobei sich sehr feste Verwachsungen innerhalb des schallleitenden Apparats bemerkbar machten. Unmittelbar nach der Operation war das Ohrensausen linkerseits verschwunden und ist auch später nicht wiedergekehrt; das Gehör dagegen scheint in diesem Falle ausnahmsweise schlechter geworden zu sein, so dass Patient bei seiner Entlassung am 11. Juli 1882 laute Sprache a. 0 . (3) nicht mehr verstand.1)

Im Mai und Juni 1884 habe ich den Kranken wiedeholentlich wiedergesehen und eingehend untersucht. Ich fand hierbei Folgendes:

L laut geschrieen (3) a. 0. wird nicht verstanden, Contra-g; c, $\mathbf{c}^{4}$, fis ${ }^{4}$ bei stärkstem Anschlag nicht gehört, Rinne -

R Fl. a. O. (3), Contra-g - $45^{\prime \prime}$ bei sehr starkem Anschlag - 60 "

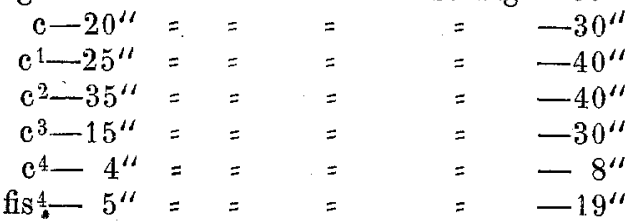

Rinne + , per Knochenleitung c vom rechten Warzenfortsatz -3 " (mit dem normalen Warzenfortsatz verglichen).

Patient hat weder jetat, noch früher jemals an Schwindel gelitten, auch nie eine Neigung dazu gespiirt; beim Stehen und Gehen mit geschlossenen Augen schwankt er nicht im Geringsten. Hält man ihm dagegen die stark angeschlagene ét-Gabel dicht vor das rechte $0 \mathrm{hr}$, so wird ihm für die Dauer einiger Secunden sehwindlig, und sehwankt er deutlich in der Richtung nach hinten und links. Seine Empfindung dabei ist folgende: "Vor den Augen wird ihm schwarz, in der rechten Kopflälfte tritt ein Druck ein, als wenn das Blut nach dem Oberkopf steigt, die Gegenstände im Zimmer drehen sich von links nach rechts im Kreise um ihn herum, und er selbst glaubt, vornüber zu fallen. Alles dieses dauert nur ganz kurze Zeit, wenige

1) Der Rinne'sche Versuch fiel hier positiv aus. Bei negativem Ausfall desselben haben wir eine Verschlechterung des Gehörs durch die genannte Operation niemals beobachtet. 
Secunden." Die Heftigkeit des Schwindels hängt sichtlich von der Stärke $a b$, mit welcher man die Stimmgabel anschlägt; bei nicht sehr starkem Anschlag bleiben die Erscheinungen ans. Achtet man während des Versuchs genan auf etwaige Bewegungen der Augäpfel, so ergibt sich, dass deren keine stattfinden, sie mî̀ssten denn so klein sein, dass wir sie objectiv nicht wahrnehmen können. Das Drehen der Gegenstände scheint vielmehr von dem unwillkürlichen Schwanken des Kranken abzuhängen. Denn wenn man ihn sitzen lässt und den Kopf fixirt, so bemerkt er selbst eine Drehung der Gesichtsobjecte nicht.

Ich habe ausser der $\mathrm{e}^{4-G a b e l}$ auch andere einwirken lassen und zwar sowohl die einen sehr lauten durchdringenden Ton gebende fis ${ }^{4}$, als anch die schwächere e- und $\mathrm{c}^{1-G a b e l . ~ U m ~ d i e ~ W i r k u n g ~}$ der beiden letzteren zu verstärken, fügte ich in den Gehörgang des Patienten die entsprechenden Resonatoren und hielt die Gabeln vor die freie Oeffnung derselben.

Einen sehr starken tiefen Ton erzeugte ich ferner durch Einschaltung eines Siemens'schen Telephons in den secundüren Stromkreis unseres Inductionsapparats. Es stellte sich heraus, dass weder fis ${ }^{4}$, noch die in genannter Weise hergestellten tiefen Töne im Stande waren, dem Kranken Schwindel zu erzeugen.

Die mitgetheilten Beobachtungen sind, wie ich glaube, für die Erklärung mancher Gleichgewichtsstörungen nicht ohne Belang. Versuchen wir sie zu verwerthen.

Zunächst wäre denkbar, dass eine stark angeschlagene, dicht vor das Obr des Kranken gehaltene $e^{4}$-Gabel das Trommelfell desselben in heftige Schwingungen versetzt, and dass die letzteren vermittelst der Gehörknöchelchenkette dem Labyrinthwasser und von diesem der Cerebrospinalflüssigkeit mitgetheilt werden, wobei durch Reizung der betreffenden Hirntheile Sehwindel entstehen könnte. Hiergegen spricht indessen, dass die bei unserem Patienten beobachteten Erscheinungen anssehliesslich durch $\mathrm{c}^{4}$, nitht aber durch fis ${ }^{\star}$, e und $\mathrm{e}^{1}$ ausgelöst werden konnten. Gerade tiefe Töne setzen, wie aus den Versuchen ron $\mathrm{Buck}$ und Burnett hervorgeht, das Labyrinthwasser in weit stärkere Bewegung als hohe.

Nun könnte allerdings $e^{4}$ in unserem Falle den Resonanzton des äusseren Gehörgangs, der ja nach v. Helmholtz in der viergestrichenen Octave liegt, darstellen und in dieser Eigenschaft besonders ausgiebige Trommelfellvibrationen erzeugen. Um hierüber ins Klare zu kommen, figte ich in das Ohr des Kranken eins der bekannten schlanchförmigen Hörrohre und liess vor dem freien ampullenartig erweiterten Ende desselben die Gabel er- 
klingen. Die schwindelerzengende Wirkung des $\mathrm{c}^{4}$ blieb auch bei dieser Anordnung die gleiche, obwohl der durch das Rohr verlängerte Gehörgang doch einen anderen Resonanzton haben musste, als der natürliche. In einer Erschütterung der Cerebrospinalflüssigkeit durch starke Trommelfellschwingungen können wir demnach die Ursache der beobachteten Gleichgewichtsstörungen nicht wohl suchen.

Ein zweiter Vorgang, an welchen demnächst zu denken wäre, ist die von $\mathrm{Hensen}{ }^{1}$ ) gefundene Contraction der Binnenmuskeln des Obres bei Einwirkung eines Schalls. Nach Hensen's Mittheilungen waren es gerade die hohen Töne, bei welchen der Tensor am stärksten zuckte, während er bei tiefen fast ruhig blieb; und ebenso verhielt es sich mit dem Stapedius. Eine heftige Zusammenziehung der genannten Muskeln bei unseren Versuchen könnte vielleicht eine starke Bewegung der Labyrinthund Cerebrospinalflüssigkeit hervorrufen und so zur Auslösung von Schwindel mitwirken.

Aber auch diese Annahme hat wenig für sich, da es doch kaum zu begreifen wäre, warum die Muskeln nur beim ${ }^{1}$, nicht aber bei dem in der Scala so nahe liegenden fis ${ }^{4}$ sich contrahiren sollten.

Der gleiche Einwand würde anch gegen den Versuch erhoben werden müssen, die beobachteten Gleichgewichtsstörungen auf eine Reizung der im Trommelfell verlaufenden Zweige des Ramus auricularis nervi vagi zu beziehen. Durch sehr starke Vibration der Membrana tympani könnten die genannteh Nerven vielleicht in Erregung versetzt werden. Indessen, wie bereits gesagt, eine Erklärung für die bei unserem Kranken beobachteten Erscheinungen wäre hiermit nicht gewonnen; denn es bliebe völlig unklar, warum nur $\mathrm{e}^{4}$, nicht aber die anderen benutzten Töne ihm Sehwindel verursachten.

Am meisten Berechtigung unter all den denkbaren Annahmen hat meiner Ansicht nach die Vorstellung, dass es bei unseren Versuchen eine Reizung des Hörnerven war, welche reflectorisch Gleichgewichtsstörungen verursachte. Dabei mag gänzlich unerörtert bleiben, ob die Erscheinungen ausgelöst wurden durch Erregung der $\mathrm{c}^{4}$-Nervenfaser, wenn ich letztere Bezeichnung brauchen darf. Möglich wäre ja auch, dass beim Erklingen der-

1) Arch. f. Physiologie. 1878, S. 312. 
$\mathrm{c}^{4}$-Gabel noch andere Theile des Acusticus, z. B. Fasern des Vestibularzweiges in Reizzustand versetzt wiirden.

Hieruber gestatten meine Beobachtungen keinen Schluss, wohl aber sprechen dieselben, wie ich glaube, mit grosser Entschiedenheit dafür, dass Reizung des Hörnerven in manchen Fällen Schwindel erzeagen kann, was Baginsky bekanntlich entschieden bestreitet. In der Literatur sind übrigens einige Mittheilungen bereits vorhanden, die Aehnliches besagen. So spricht z. B. Roosa ${ }^{1}$ ) von einer Sängerin, die Schwindel bekam beim Singen eines hohen Tones, Bechterew ${ }^{2}$ ) von einer Person, bei welcher Gleiches eintrat infolge des Geräusches, welches ein im Schnee gleitender Schlitten verursacht, und von einer zweiten, welche aus demselben Grunde das Gẹräusch auf einem sandigen Wege rollender Räder nicht vertragen konnte.

Von Interesse ist in Fall 10 ferner eine Erscheinung, auf welche ich im XIX. Bd. (S. 45) dieses Archivs bereits aufmerksam gemacht habe, nämlich eine vorübergebende, Ermü dung" des Ohres bei Einwirkang starker Töne. Dieselbe ist bei diesem Patienten ausserordentlich eclatant, die Differenz der Hörzeit zwischen seinem und einem normalen $\mathrm{Ohr}$ eine vollkommen verschiedene bei leisem und bei stärkerem Anschlag der Gabel, wie das aus den vorhergehenden Notizen hervorgeht. Wie diese Erscheinung hier zu erklären ist, vermag ich nicht zu sagen.

Unter den mit Pilocarpin behandelten Kranken befanden sich drei, welche ihr Ohrenleiden auf eine traumatis che Einwirkung zuriuckführten. Zwei dieser Fälle, welche mir in mancher Beziehung bemerkenswerth erseheinen, lasse ich hier folgen.

11. Ludwig Krause, 42 Jahre alt, Stuckateur aus Berlin, stürzte am 22. Oetober 18812 Meter hoch vom Gerüst auf Steinstufen. Mehrere Stunden blieb er besinnungslos. Als das Bewusstsein zuritckkehrte, bemerkte er rechterseits Schwerhörigkeit und permanentes gleichmässiges Sansen. Gleich nach dem Sturz soll etwas Blut aus dem rechten Ohre geflossen sein. Nachdem er 14 Tage lang in der chirurgischen Abtheilung der Charité gelegen hatte, stellte er sich am 9. November 1881 in der otiatrischen Poliklinik zur Untersuchung. Hier fanden wir im rechten Gehörgang etwas angetrocknetes Blut, sonst aber beiderseits normalen Ohrenspiegelbefund.

1) Zeitschrift f. Ohrenheilk. IX. Bd. S. 338.

2) Pflüger's Archiv. XXX. Bd. S. 343. 
R Fl. $0,3(3), c-0^{\prime \prime}, c^{4}$ und fis $\mathbf{4}^{-20}$, e bei Application der Gabel auf den rechten Warzenfortsatz $-10^{\prime \prime}$ (mit dem normalen Warzenfortsatz verglichen $\left.{ }^{1}\right)$ ), Rinne + .

c wird per Kopfknochenleitung nicht nur vom Scheitel, sondern sogar von der linken Schädelhälfte aus nach rechts gehört.

L Fl. 6 (Friedrich).

Seit dem Fall permanentes Sausen, mitunter auch Schwindel.

Catheterismus rechts: normales Auscultationsgeräusch, keine Veranderung des Gehörs und des Sausens.

Wir verordneten Kal. jodat. und Kal. bromat. innerlich. Eine Besserung indessen erfolgte nicht, und daher wurde Patient am 31. Januar 1882 in die Klinik aufgenommen, wo er bis zn seiner Entlassung am 9. Februar 18826 subcutane Pilocarpininjectionen (à 0,01 Grm.) erhielt. Anch hierdurch ist eine Besserung nicht erzielt worden.

Von Interesse ist in dem vorliegenden Fall das Ergebniss der Stimmgabelprüfung. Höchst auffallend ist zunächst die eclatante Verschiedenheit der Hörschärfe für hohe und tiefe Töne, insofern das tiefe c wie ron einem normalen Ohr bis $\mathrm{zum}$ völligen Verklungensein, das hohe durchdringende $\mathrm{c}^{4}$ und fis ${ }^{4}$ dagegen ganz ausserordentlich schlecht gehört wurde.

Ich glaube wohl, dass wir auf Grund dieses eigenthïmlichen Verhaltens eine Erkrankung des schallempfindenden Apparates anzunehmen berechtigt sind.

Sehr eigenthümlich ist ferner der Ausfall der Untersuchung per Kopfknochenleitung. Der Rinne'sche Versuch war positiv; das spräche also für eine Affection des inneren Ohres.

Vom Scheitel dagegen, ja sogar von der linken Kopfhälfte aus wurde $c$ anf dem kranken rechten Ohre stärker gehört; demnach müsste also nach Ansicht vieler Autoren eine Mittelohrerkrankung angenommen werden.

Dieser Widerspruch würde gelöst sein, wenn wir nicht nur den schallempfindenden, sondern auch den schalleitenden Apparat als afficirt betrachten wollten. Indessen hierfur liegt gar keine Veranlassung vor. Die objective Untersuchung ergab normale Verhältnisse, nach Application der Luftdouche (Katheter) änderte sich weder Sohwerhörigkeit, noch Sausen.

Wenn man dieses Alles unbefangen erwägt, so wird man sich, glaube ich, kaum der Ansicht erwehren können, dass eine Ver-

1) Zu diesem Vergleiche benutzte ich mein eigenes Ohr. Wenn der Patient den Ton vom Warzenfortsatz aus mehrere Secunden kürzer hörte, so liegt das vielleicht daran, dass er über 12 Jahre älter war, als ich. 
stärlung der Perception von den Kopflknochen aus nicht nur, wie die meisten Autoren behaupten, durch Erkrankung des Schallleitungsapparates, sondern auch durch pathologische Veränderungen im Labyrinth oder andëre bisher noch unbekannte Verhältnisse bedingt sein kann.

12. Dem 22 Jahre alten Schlosser Reinhold $J_{a} \mathrm{cob}$ ans Berlin fiel am 19. November 1881 eine Geriststange anf den Kopf, wodurch or eine "Gehirnerschütterung und Fractur des rechten Felsenbeins " erlitt. Die ersten 4 Wochen war Patient besinnungslos; nach der Wiederkehr des Bewusstseins bemerkte er sofort rechtsseitige Taubheit und gleichmässiges Ohrensausen. Beim Gehen schwankte er von einer Seite zur anderen, und zwar im Hellen ebenso, wie im Dunkeln. Im Sehvermögen beobachtete er keine Veränderung; auch hat er trotz der bestehenden Gleichgewichtsstörungen niemals eine Scheinbewegung der in seiner Umgebung befindlichen Gegenstände und ebensowenig eine Zunahme des Schwindelgefühls bei raschen Bewegungen wahrgenommen. Das Sansen blieb unverändert stark, der Schwindel dagegen verringerte sich allmählich, so dass sich einige Monate nach Beginn des Leidens täglich nur 2-3 Anfälle von etwa 15 Minuten Dauer einstellten, bei denen Patient, wenn er gehen wollte, wie ein Betrunkener taumelte. Am 28. Februar 1882 liess er sich in die Klinik aufnehmen. Hier fanden wir rechts im vorderen unteren Trommelfellquadranten eine dreieckige Narbe; der Hammergriff schien abnorm weit nach vorn zu liegen und weit aus dem Niveau des Trommelfells herauszuragen, so dass er fast wie luxirt aussah.

L Fl. 2 (Kakadu).

R Fl. a. 0. nicht gehört, Contra-g-, c-, cL- und fis4-Gabel bei stärkstem Anschlag nicht, $c$ und $\mathrm{c}^{1}$ nicht einmal bei Zuhülfenahme der betreffenden Resonatoren, $\mathrm{c}^{2}-35^{\prime \prime}, \mathrm{c}^{3}-12^{\prime \prime}, \mathrm{e}^{4-}-8^{\prime \prime}$, c per Knochenleitung vom Scheitel nach links gehört.

Catheterismus rechts normales Auscultationsgeräusch; danach Sausen stärker, Gehör unverändert. Patient erhielt in der Klinik subcutane Pilocarpininjectionen (à $0,01 \mathrm{Grm}$.), und zwar bis zu seiner Entlassung am 13. März 1882 im Ganzen 8, indessen besserte sich weder das Sausen, noch das Gehör.

Auch in diesem Fall lieferte die Stimmgabelprïfung ein interessantes Resultat, insofern hier nur der mittlere Theil der Scala $\left(\mathrm{c}^{2}, \mathrm{c}^{3}, \mathrm{c}^{4}\right)$ noch gehört wurde, während die Perception der höchsten und tiefsten Töne vollkommen verloren gegangen war. Wir schliessen hieraus auf eine Erkrankung des inneren Ohres.

$\mathrm{Zu}$ der Zahl derjenigen Kranken, welchen die Pilocarpineur wenigstens einigen Nutzen gebracht hat, gehört auch eine Patientin, welche anscheinend im Verlauf einer Meningitis ihr Hörver- 
mögen eingebüsst hatte. Ausser diesem unter Nr. 5 bereits mitgetheilten Fall sind in der Klinik in gleicher Weise noch vier andere behandelt worden, bei welchen das Ohrenleiden auf ähnliehe Art entstanden war.

Es wäre zwecklos, die betreffenden Journalnotizen hier in extenso wiederzugeben, nachdem von Moos ${ }^{1}$ ) allein mehr als 60 derartige Fälle beschrieben worden sind. Ich nehme hiervon um, so eher Abstand, als unsere Aufzeichnungen, so weit sie die acute Erkrankung betreffen, sich nicht sowohl auf eigene Beobachtung oder genaue Berichte seitens der behandelnden Aerzte, als vielmehr auf die gewiss häufig unzuverlässige und unzureichende Schilderung der Angehörigen stützen, ein Umstand, weleher, wie auch Moos hervorhebt, unserer Erkenntniss der in Rede stehenden Affection ausserordentlich im Wege steht. Aus diesen Gründen sei hier nur kurz berichtet, dass von den erwähnten vier Fällen der eine im 2., einer im 18. und zwei im 8. Lebensjahre standen, als sie von einer anscheinend unter dem Bilde der Meningitis verlaufenden Erkrankung befallen wurden, dass Taubheit bereits in den ersten Tagen eintrat, und dass mit Ausnahme der im 18. Jahre erkrankten Patientin alle auch nach der Genesung noch längere Zeit hindurch taumelnden Gang zurückbehielten. In einem Falle kehrte etwa ein halbes Jahr nach Eintritt der Meningitis spontan eine Spur vom Gehör zurüek, alle anderen blieben taub, und die in der Klinik eingeleitete Behandlung erwies sich bei ihnen vollkommen erfolglos.

Hiermit sind die Mittheilungen, welche ich beztiglich der einer Pilocarpincur unterworfenen Kranken zu machen hätte, zum Abschluss gelangt. Es sei mir gestattet, die gewonnenen therapeutisehen Ergebnisse einer kurzen Bespreehung zu unterziehen.

Unter 35 Kranken, welche in der genannten Weise behandelt wurden, hat sich bei 24 die Hörschärfe für Sprache in keiner Weise gebessert, bei 6 trat eine geringfügige, kaum nennenswerthe, bei 5 anderen allerdings eine bedeutende Zunahme des Hörvermögens ein. Das Gesammtresultat ist demnach immerhin ein ziemlich ungünstiges zu nennen. Nichtsdestoweniger dürften sich weitere Versuche mit Pilocarpinbehandlung empfehlen, und zwar nicht nur in solehen Fällen, in welchen wir die Diagnose einer Nerventaubheit stellen. Es gibt eine Reihe von Schwerhörigen, bei denen weder die Untersuchung mit versehieden hohen

1) Ueber Meningitis cerebrospinalis epidemica. Heidelberg 1881. 
Stimmgabeltönen, noch der Rinne'sche Versuch oder eine andere Art der Hörprüfung per Kopfknochenleitung sicheren Anhalt für die Annahme eines Hörnervenleidens bieten, in denen aber die Bebandlung mit Luftdouche, bez. medicamentösen Injectionen in die Paukenhöhle per Katheter entweder gar nichts hilft, oder nur bis zu einem gewissen Grade Besserung herbeiführt, nm bei weiterer Foxtsetzung ihre Dienste zu versagen. In diesen Fällen, wo uns therapentisch höchstens operative Eingriffe, an ihrer Spitze die von Lucae neuerdings empfohlenen "Stempelbewegungen", uibrig blieben, ist auch der vorsichtige Versuch einer Pilocarpincur nach meiner Ansicht statthaft. Und ich halte es für sehr wohl möglich, dass gerade bier mit dieser Behandlung gïnstigere Erfolge erzielt werden würden, namentlich wenn wir dieselbe nicht auf veraltete, weit vorgeschrittene Ohrenleiden beschränken. In der Klinik freilich werden solche Erfahrungen schwer zu gewinnen sein, da sich Patienten, deren Schwerhörigkeit nur mässigen Grades ist, zur Aufnahme kanm entschliessen.

Wenn ich nunmehr zu anderen bemerkenswerthen Fällen übergehe, so wäre zunächst eines Kindes zu gedenken, bei welchem rasches ohrenärztliches Eingreifen noch in letzter Stunde drobende Lebensgefahr abwandte.

12. Sehwere meningitische Erseheinungen im Verlauf einer chronischen eitrigen Mittelohrentziindung. Heilung dureh Entfernung von Eiter zuriickhaltenden Polypen.

Marie Siebke, 10 Jahre alt, Maurerstochter aus Berlin, wird am 30. Januar 1883 in soporösem Zustande in die Klinik gebracht. Seit 4 Jahren soll das linke $\mathrm{Ohr}$ eitern und zeitweise schmerzen. Drei Wochen vor der Aufnahme sistirte der Ausfluss einige Tage hindurch, und bestand während dieser Zeit neben starkex Röthung, Schwellung und Druckempfindlichkeit der linken Warzenfortsatzgegend so heftiges Ohren - und Kopfreissen, dass Patientin das Bett nicht verlassen konnte. Die nächsten 14 Tage hat sie sich wieder völlig wohl befunden. Am 29. Januar traten Schmerzen in der linken Kopfhälfte, bald darauf mehrmaliges Erbrechen und bereits nach wenigen Stunden Bewusstlosigkeit ein. In der folgenden Nacht hat sie häufig, namentlich bei Berihrung laut geschrieen und mit den Zähnen geknirscht. Stuhl und Urin wurden ins Bett entleert.

24 Stunden nach der Erkrankung erfolgte die Anfnahme in die Klinik, wo wir Sopor, Nackenstarre, Zähneknirschen, Crie hydrencéplalique, kahnförmig eingezogenen Leib, starke Hyperästhesie der Hant, 390 Körpertemperatur und eine Pulsfrequenz von 84 constatirten. Im linken Obre stinkender Eiter und ein grosser Polyp, welchen Prof. L u c a e sofort entfernte; dann wurde mit $21 / 2$ proc. Carbolsäurelösung. ausgespritzt, wobei die Fliissigkeit durch die Tuba abfloss, Eisblase 
auf den Kopf gelegt und ein Laxans von Calomel und Jalape verabreicht.

12 Stunden nach der Entfernung des Polypen stellte sich wieder Bewusstsein und sehr bald fast vollkommenes Wohlbefinden ein, welches nur zeitweise durch leichte Kopfschmerzen getrübt wurde. Das linke $\mathrm{Ohr}$ eiterte stark.

L Fl. $0,4(3)$.

R Fl. normal.

e von der Mittellinie des Kopfes per Knochenleitung beiderseits gleich gut gehört.

Am 5. Februar zeigt sich hinter dem linken Ohr ein etwa mandelgrosser, verschieblicher, nicht druckempfindlicher Tumor; derselbe wird mit Pravaz'scher Spritze punctirt; es entleeren sich hierbei nur einige Tropfen Blut.

Am 19. Februar entwickelt sich, nachdem wieder heftigere, mehrere Stunden anhaltende Kopfschmerzen und eine Temperatursteigerung bis $39,4^{0}$ vorausgegangen waren, über dem linken Warzenfortsatz eine stark geröthete, bei Beriuhrung schmerzhafte Anschwellung. Bei Druck anf dieselbe quillt Eiter aus dem Gehörgang. Der letztere erscheint durch eine Schwellung der hinteren oberen Wand verengt. Die Geschwulst auf dem Warzenfortsatz wird incidirt; in der Tiefe der Wunde erscheinen missfarbige Granulationen; bei Sondenuntersuchung fühlt man cariösen Knochen. Einführung eines Drains. Verband mit Jodoformgaze. Unmittelbar nach der Operation Abfall der Temperatur zur Norm.

Am 24. Februar werden in der Chloroformnarkose die Granulationen aus der auf dem Warzenfortsatz angelegten Incisionswunde entfernt; hierbei gelangt man in einen tiefen Fistelkanal, welcher drainirt wird. Beim Ausspritzen des Gehörgangs fliesst das Spritzwasser in starkem Strahl ans dem Drain heraus.

Am 6. April Entlassung aus der Klinik. Schmerzen und Druckempfindlichkeit in der Regio mastoidea sind nicht wiedergekehrt, die Ohreneiterung hat bereits seit langer Zeit bedeutend nachgelassen.

R Fl. normal, L Fl. 1,5 (3).

In diesem Falle kam Hülfe noch zur rechten Zeit. Wäre der den Gehörgang verlegende Polyp, durch welchen Eiterretention in der Tiefe bedingt wurde, noch länger im Ohre verblieben, so wïrde das Kind seinem consecutiven Hirnleiden unzweifelhaft erlegen sein.

Ob letzteres als Meningitis zu bezeichnen ist, oder ob wir es nur mit Hirnhyperämie zu thun hatten, ist eine Frage, welche gegentiber der eclatanten praktischen Wichtigkeit des vorliegenden Falles von unwesentlicher Bedeutung zu sein scheint. War eine purulente Entzündung der Hirnhäute vor Aufnahme des Kindes in die Klinik noch nicht erfolgt, so hätte sie sich doch sicher in kurzer Zeit entwickelt, wenn nicht zuvor dem im Mittel- 
ohr zurïckgehaltenen Eiter durch Entfernung des Polypen freier Abfluss geschafft worden wäre.

Der glückliche Ausgang zeigt wiederum, dass die geeignete otiatrische Behandlung selbst da noeh lebensrettend wirken kann, wo infolge der Ohreneiterung bereits schwere Erkrankung des Gesammtorganismus sich ausgebildet hatte.

Leider ist unser therapeutisches Handeln nicht immer von gleich günstigem Erfolge gekrönt. Das beweisen drei in der Klinik beobachtete Fälle, in welchen wir den tödtlichen Ausgang nicht hindern konnten. Auch bei diesen war das ursächliche Leiden eine chronische eitrige Entziundung des Mittelohres, welche bei einer Kranken zur Pyämie, bei zwei anderen zu purulenter Meningitis fuhrte. Nor in einem dieser Falle hatten wir einen grösseren operativen Eingriff, die Aufmeisselung des Warzenfortsatzes, unternommen, in der Hoffnung, hierdurch eine Heilung: noch herbeiführen za können. Indessen das. Hirnleiden war bereits zu weit gediehen, und der Tod war nicht mehr abzuwenden.

Ich bemerke an dieser Stelle, dass unter den 31 klinischen Kranken, bei welchen Warzenfortsatzoperationen in den ersten 3 Jahren ausgeführt worden sind, ein weiderer Todesfall nicht $z u$ verzeichnen ist. Die übrigen verliefen günstig, indessen ist bei einzelnen von ihnen die Nachbehandlung noch nicht beendet, und somit ein endgültiges Urtheil über den Ausgang zur Zeit nicht abzugeben. Dieses ist der Grund, warum in vorliegenden Bericht die zahlreichen Fälle, in denen die chirurgisehe Eröffnung des Warzenfortsatzes vorgenommen wurde, nicht aufgenommen worden sind.

Was nun die anderen beiden Fälle anlangt, in welchen Exitus letalis eintrat, so waren es folgende:

13. Chronisehe eitrige Mittelohrentzündung. Caries. Tod an Pyämie.

Fran Weise, 29 Jahre alt, Arbeitersfrau aus Gorka (Posen). A ufnahme in die Klinik am 11. Januar 1883.

Die Kranke leidet seit frïhester Kindheit an linksseitiger Otorrhoe. Vor 8 Wochen traten Fieber und in dem linken Ohre Schmerzen ein, die häufig so heftig waren, dass Patientin Nachts nicht schlafen konnte. Dabei war dem Ausfluss öfters Blat beigemischt.

Am 6. Januar ein starker Schüttelfrost, der sich nach zwei Tagen wiederholte.

In der Klinik fanden wir rechts normalen Spiegelbefund, links einen grossen Polypen, der den Gehörgang ziemlich ausfüllte. Nach Entfernung desselben mit der Wilde'schen Schlinge entleeren sich beim Ausspritzen des Ohres viele übelriechende eingedickte Eiterbröckel. 
Rechts ist Patientin ungewöhnlich feinhörig, links wird Fl. (3) a. 0. bei geschlossenem und geöffnetem Gehörgang gleich gut, c bei stärkstem Anschlag nicht gehört, fis ${ }^{4}-8^{\prime \prime}$, Rinne -

c wird per Kopfknochenleitung vom Seheitel sowohl, wie von der Stirnmitte aus nach links gehört.

Kein Ohrensausen. Puls 120. Temperatur Morgens 39,2 ${ }^{\circ}$, Abends $39,6^{0}$. Ordination: Ausspritzen des Ohres mit 21/2 proc. Carbolsäurelösung.

12. Januar. Nacht unruhig, aber ohne Schmerzen. 10 Uhr Vormittags starker Schittelfrost (410 Temperatur). Linker Tragus und Warzenfortsatz druckempfindlich. Untersuchung der Brust- und Bauchorgane ergibt bis auf eine Vergrösserung der Milz und Albuminurie normalen Befund. An den Augen nichts Abnormes. Puls 94. Abendtemperatur $37,7^{\circ}$.

13. Januar. Nacht schlaflos. Die Spitze des linken Warzenfortsatzes und die darunter gelegene Partie des Halses stark druckempfindlich. Puls 120. Temperatur Morgens 37,00, Abends 38,50.

14. Januar. Nacht wegen starker Sehmerzen in der linken Seite des Halses schlaflos. Die Spitze des linken Warzenfortsatzes und die Gegend unterhalb desselben, zwischen dem Ansatz des M. sternocleido-mastoideus und der Wirbelsäule äusserst druckempfindlich. Puls 136. Temperatur Morgens 38, $5^{0}$, Abends $40,3^{\circ}$.

15. Januar. Nacht schlaflos; Schmerz in der ganzen linken Kopfseite. Der Kopf wird nach links gewandt gehalten, Drehung desselben nach rechts verursacht Schmerzen. Beim Ausspritzen des Ohres werden ïbelriechende käsige Massen and ein kleiner Polyp herausgespiult. c per Knochenleitung von der Mittellinie des Kopfes aus nach links gehört. Icterische Verfärbung der Conjunctiven. Puls 124, Temperatur Morgens 40,30, Abends Schïttelfrost $\left(40,6^{\circ}\right)$. Ordination: Abends Chinin. muriat. 0,5.

16. Januar. Nacht schlaflos; seit Morgen sind die Schmerzen in der linken Seite des Halses geringer, dagegen sind sehr starke Schmerzen an der Halswirbelsänle anfgetreten. Icterische Hautfärbung. Beim Spritzen entleeren sich viel cholesteatomatöse Massen. Das rechte $\mathrm{Ohr}$, welches bisher absolut normal functionirte, ist seit heute etwas schwerhörig.

R Fl. 2 (3) 1 (Bismark), a. 0. (Berlin).

Die Spiegeluntersuchung ergibt geringe Injection der Hammergriffgef ässe, sonst nichts Abnormes.

Nachdem Patientin Mittags 0,5 und Abends noch 1,0 Grm. Chinin. muriat. genommen hat, hört sie R Fl. 0,6 (3), 0,3 (Bismark, Berlin), $\mathrm{c}-30^{\prime \prime}, \mathrm{e}^{4-6} 6^{\prime \prime}, \mathrm{fis}^{4}-5^{\prime \prime}$.

c per Knochenleitung von der Mittelliniedes Kopfes aus nach links gehört. Puls 128. Temperatur Morgens 38,30, Abends $40,2^{\circ}$.

17. Januar. Nacht schlaflos. Warzenfortsatz viel weniger druckempfindlich als frïher. Puls 128. Temperatur Morgens 39,5, Abends 39,5 . Ordination: Chinin. muriat. 0,25 dreistiundlich.

18. Januar. Nacht schlaflos. e per Knochenleitung von 
der Mittellinie und von den meisten anderen Punkten des Kopfes, so auch von dem rechten Warzenfortsatz aus nach links gehört, von einzelnen Punkten der Mittellinie, z. B. von der Stirnmitte aus, wird c heute auch bei stärkstem Ansehlag gar nicht mehr gehört. Gesicht etwas eyanotisch, 72 Athemzïge in der Minute, Puls 152, klein.

Temperatur Morgens $40,3^{\circ}$, Abends $40,6^{\circ}$.

Abends $61 / 2$ Uhr erfolgt der Tod.

Section (Dr. Israel): Stark icterische Färbung der ganzen Haut. Am Schädeldach alle Nähte verknöchert, sonst keine Abweichung. Dura und pia mater stark icterisch. An der Schädelbasis zeigt die Dura in der Umgebung des Porus acust. int. sinister viele zarte Vascularisationen. Nachdem man die Dura von der linken Pars petrosa abgezogen hat, bemerkt man an der letzteren einen etwa $6 \mathrm{Mm}$. in Quadrat messenden Verknöcherungsdefect, in dem die Schleimbaut des Mittelohres in directer Verbindnng mit der Dura gestanden hat, und der genau der Stelle des Tegmen tympani entspricht. Von hier aus erstreckt sich an der hinteren Seite der Pars petrosa ein, wie es scheint, cariöser Knochendefect, der durch etwas missfarbige, bröcklige Eitermassen ausgefülltt erscheint. Der Sinus transversus vollkommen intact; es findet sich in ihm ein etwa $3 \mathrm{Mm}$. langer icterisch gefärbter Thrombus, welcher einen vollkommenen Abschluss an der Stelle des Ueberganges in den Knochen herbeigefithrt hat. Die linke Vena jugularis sowohl in ihrer Wand jauchig infiltrirt, als auch umgeben von einer aus dem Knochen fortgesetzten jauchigen Infiltration, die in directem Zusammenhang mit dem Herd des Felsenbeins steht. Ein kleiner circa linsengrosser Verknöcherungsdefect findet sich an der hinteren Seite der rechten Pars petrosa. Von der linken Vena jugularis und dem infiltrirten Gewebe des Hinterhaupts aus erstreckt sich nach der Pars condyloidea des Atlas eine geringfiugige jauchige Infiltration, welche nach einer kleinen cariösen Stelle des Bogentheils vom Atlas führt. In der Lunge sehr zahlreiche jauchige metastatische Abscesse. Frischer pulpöser Milztumor. Parenchymatöse Nephritis und Hepatitis.

Die von Herrn Prof. Lucae ausgeführte Untersuchung des linken Gehörorgans ergab Folgendes:

Der Acusticus bis zum Tractus foraminulentus sowie der Facialis zeigen mit Ausnahme einer sehwachen Röthung des Neurilems normales Verhalten. . Ein erbsengrosser, dreieckig gestalteter Verknöcherungsdefect nach binten von der Paukenhöhlendecke gelegen in der Gegend des oberen Kanals. Von diesem nichts zu sehen. Nach hinten geht der Verknöcherungsdefect in einen an der hinteren Seite des Felsenbeines gelegenen, unregelmässig runden cariösen Krater von $1 \frac{1 / 2}{\mathrm{Cm}}$. Durchmesser über. Der letztere nach innen nur $2 \mathrm{Mm}$. von dem Porus acust. int., nach hinten ca. $1 \mathrm{Cm}$. von der Fossa sigmoidea entfernt, schickt einen kleinen Fortsatz nach unten, welcher an der Umknickungsstelle des Sinus transvers. die Vena jugularis direct trifft. Der ganze so gebildete Krater ist mit fauligen Granulationsmassen resp. Detritus gefüllt, welche sich durch ein an 
der oberen Wand des Bulbus venae jugularis befindliches Loch in denselben fortpflanzen.

Aus der Beschreibung erhellt, dass auch der hintere halbzirkelförmige Kanal durch Bildung des Kraters zerstört worden ist. Nach Aufbruch des inneren Obres pflanzt sich der letztere sowohl in den Vorhof, wie in die Schnecke fort, welche Theile durchweg mit Granulations- und käsigen Massen angefüllt sind. Zwischen diesen findet sich als einziges Ueberbleibsel der Schnecke ein nekrotischer Rest der Lamina spiralis, bei genauer mikroskopischer Untersuchung keine Spur von normalem Gewebe. In der Gegend des ovalen Fensters ein entsprechendes Loch, durch welches man mit der Sonde direct in die Paukenhöhle gelangt. Oberhalb noch ein Defect. Keines von den Gehörknöchelchen vorhanden. Hintere Wand des knöchernen Gehörgangs fehlt vollständig.

Es handelte sich in dem vorliegenden Falle um eine tödtlich verlaufende Pyämie, deren Ausgangspunkt wir in der linksseitigen chronischen Mittelohreiterung zu suchen haben. Dass eine Aufmeisselung des Warzenfortsatzes hier noch etwas genitzt hätte, ist kaum anzunehmen. Herr Prof. Lucae verzichtete anf djeselbe, da er sich angesichts der bereits wochenlang dauernden schweren Allgemeinerscheinungen einen Erfolg von der Operation nicht mehr versprach.

Von besonderer Wichtigkeit erscheint mir in der mitgetheilten Krankengeschichte, dass die Patientin während ihres ganzen Aufenthalts in der Klinik bis kurz vor Eintritt des Todes die c-Gabel von der. Mittellinie des Kopfes aus ausschliesslich im kranken Ohre hörte; und zwar sang sie auf meine Aufforderung den Ton der Gabel richtig nach; wodurch ibre Angabe noch an Zuverlässigkeit gewinnt. Ich bemerke hierzu, dass die Kranke äusserst intelligent, bei der Untersuchung von grosser Aufmerksamkeit und immer bei klarem Bewusstsein war, so dass nach meiner Ansicht ihre Aussagen unbedingten Glauben verdienen. Wie sollen wir nun aber letztere mit dem Sectionsbefund in Einklang bringen?

Das innere Ohr mit Ausnahme des Acusticusstammes war linls durch Eiterung zerstört, und doch nahm Patientin den Ton der Gabel vom Schädel aus auf dieser Seite stärker wahr, als auf der gesunden rechten.

Es lässt sich nicht leugnen, dass diese Thatsache in unversöhnlichem Widerspruch steht $\approx u$ der von den meisten Autoren angenommenen und auch in den besten Lehrbüchern vertretenen Lehre von dem differentialdiagnostischen Werth der Stimmgabelprüfung von den Kopfknochen aus. Ich erinnere daran, dass 
auch in Fall 11 unser Prüfungsergebniss mit den herrschenden diesbezüglichen Ansichten schwer in Einklang zu bringen war. Derartige Beobachtungen scheinen mir die angebliche Bedeutung der genannten Untersuchungsmethode stark zu erschüttern. In diesem Sinne ist hier auch der von Christinneck ${ }^{1}$ ) beschriebene, von Herrn Prof. Schwartze selber untersuchte, bemerkenswerthe Fall zu erwähnen, bei welchem trotz Ausstossung der nekrotischen rechten Schnecke die auf den Scheitel gesetzte C-Gabel rechts stärker gehört wurde.

Herr Prof. $\mathrm{Lucae}^{2}$ ) hat bereits vor vielen Jahren auseinandergesetzt, dass die Prüfung der Stimmgabelperception von den Kopfknochen aus für die Diagnostik der Ohrenkrankheiten im Allgemeinen fast bedeutungslos sei. Er gestand der genannten Untersuchungsmethode einen Werth nur in den Fällen zu, in welchen im Verlauf einseitiger acuter Mittelohreiterungen drohende Gehirn- und Allgemeinerscheinungen auftreten. Hier hielt er eine ernstere Ohraffection und ein Uebergreifen derselben auf das Gehirn mit grosser Wahršcheinlichkeit für ausgeschlossen, so lange die Stimmgabel vom Schädel aus constant auf dem leidenden Ohre stärker percipirt wurde. Er stiutzte diese Annahme auf seine an zahlreichen derartigen Kranken gewonnenen praktischen Erfahrungen.

Durch den soeben mitgetheilten Fall 13 werden die letzteren modificirt, insofern nunmehr bewiesen ist, dass auch da, wo die Stimmgabel per Kopfknochenleitung auf der kranken Seite stärker gehört wird, vom Ohre aus tödtlicher Ausgang erfolgen, und dass das Labyrinth hier vollkommen zerstört sein kann.

Herr Prof. Lucae hat sich seitdem in seinen klinischen Vorträgen dahin ansgesprochen, dass man nun, wenn der Ton einer auf den Schädel aufgesetzten Stimmgabel auf dem kranken Ohre stärker gehört wird, als auf dem gesunden, hieraus nichts Anderes mehr schliessen könne, als dass der Stamm des Hörnerven im Wesentlichen gesund sei.

14. Peter Merken, 31 Jahre alt, Schutzmann aus Berlin, sucht am 24. November 1882 die Poliklinik auf. Patient leidet seit 6 Tagen an sehr heftigen Schmerzen und geringer Eiterung im linken Ohre, hat deshalb die letzten 6 Nächte nicht mehr schlafen können und bereits seit 4 Tagen das Bett gehütet.

1) Dieses Archiv. XVIII. Bd. S. 294:

2) Die Schallleitung durch die Kopfknochen und ihre Bedeutung für die Diagnostik der Ohrenkrankheiten. Würzburg 1870. 
Die Untersuchung ergibt eine eitrige Mittelohrentzündung mit Perforation des Trommelfells.

L Fl. $0,3(3)$, c bei stärkstem Anschlag nicht, per Knochenleitung vom linken Warzenfortsatz aus fast bis zu Ende, $0^{4}$ mässig, fis 4 sehr schlecht gehört.

Tragus und Warzenfortsatz nicht druckempfindlich. Auf letzterem mehrere alte Schnittnarben. Patient hat 1876 bereits 4 Monate lang wegen linksseitiger Ohreneiterung im Lazareth gelegen, und ist damals eine Anschwellung hinter der Ohrmuschel mehrmals incidirt worden. Ordination: Borsäure zum Ausspritzen des Ohres und hydropathische Ueberschläge auf dasselbe.

In der Klinik zu bleiben, wozu ihm dringend gerathen wird, kann sich Patient nicht entschliessen.

Am 2. December Abends wird er in schwer krankem Zustande aufgenommen. Wir erfahren, dass er am 24. November, aus der Poliklinik zurtickgekehrt, einen Schüttelfrost von einstïndiger Dauer, am 25. zwei und am 27. wiederum einen derartigen Frostanfall gehabt hat. Die Obrenschmerzen haben unter Anwendung der hydropathischen Umschläge rasch nachgelassen, dagegen traten am 28. ansser häufigem Erbrechen und hartnäckiger Stuhlverstopfung sehr heftige Schmerzen im Oberkopf ein, so dass der Kranke Tag und Nacht wimmerte. Am 29. leichte Delirien und lallende Sprache.

In der Klinik erscheint sein Bewusstsein etwas getrübt; er gibt auf Fragen nur langsam Antwort. Lähmungserscheinungen sind nicht zu constatiren. Puls 76. Abendtemperatur 38 ${ }^{\circ}$. Der linke Warzenfortsatz äusserlich normal, seine Spitze, sowie die Basis des Hinterhaupts druckempfindlich. Ordination: Eisbentel, Klysma, Blutegel auf die druckempfindlichen Stellen hinter dem Ohre, Ausspritzen des letzteren mit Borsäurelösung.

3. December. Sopor, Incontinentia alvi et urinae. Temperatur Morgens $37,5^{\circ}$, Abends $37,8^{\circ}$.

4. December. Pupillen sehr eng und reactionslos. Cornea anästhetisch. Contracturen der Extremitäten. Sehr ausgesprochenes Fussphänomen. Kniephänomen normal. Hautreflexerregbarkeit erscheint erlosehen. Stokes'sche Athmung.

Temperatur Morgens 36,90, 2 Uhr Nachmittags Tod.

Section nicht gestattet.

Unsere Diagnose lautete hier: "Meningitis purulenta infolge ron chronischer eitriger Mittelohrentzündung."

Sowohl im letzten wie auch im vorletzten Fall büssten die Kranken die Vernachlässigung ibres Ohrenleidens mit dem Tode. Hätten sie rechtzeitig eine otiatrische Behandlung nachgesucht, so hätte die Eiterung vielleicht beseitigt, und ein Uebergreifen des Processes auf das Gehirn und die grossen Blutleiter verhätet werden können.

Wenn manche Aerzte trotzdem eine Scheu haben, chronische Ohrenflüsse in Behandlung zu nehmen, so liegt der Grund hier- 
für, wie das v. Tröltseh in seinem Lehrbuch in klassischer Weise ausfubrt, zum Theil in der Erfabrung, dass alte Otorrhoiker, die bisher von ihrem Leiden vielleicht niemals eine Beschwerde hatten, den lobenswerthen Entschluss, sich einer specialistischen Cur zu unterwerfen, nicht selten mit heftigen Schmerzen entgelten müssen und zwar einfach deshalb, weil beim Ausspritzen in den Hoblräumen des Mittelohrs eingetrocknete Secretmassen aufquellen und dann durch Druck anf die Nachbarschaft entzündungserregend zu wirken beginnen.

Ein ähnlicher Vorgang, wie der eben angedeutete, fand in dem folgenden Falle statt:

15. Chronisohe eitrige Mittelohrentzïndung mit Bildung von Polypen, Entfernung der letzteren mit dem seharfen Löffel. Pyïmie? Heilung.

Anna Rossberg, 19 Jahre alt, Schneiderin aus Berlin. A u f nahme in die Poliklinik am 1. A ugust 1883. Patientin hat in der Kindheit häufig Ohrenreissen gehabt, seit einem Vierteljahr leidet sie rechts an Ohreneiterung, Sausen und Schwerhörigkeit. Im rechten Gehörgang polypöse Massen, von denen am 1. und 15. August ein Theil mit der Wilde'schen Sohlinge entfernt wird.

Am 17. Angust 1883 Aufnahme in die Klinik, wo am nächsten Tage wiederum einige Polypen abgetragen werden. Da letztere indessen immer wieder rasch nachwuchsen, so wird Patientin am 24. August chloroformirt, und nun in der Narkose mit dem scharfen Löffel eine gründliche Entfernung der Wucherungen vorgenommen. Hierbei gelangt man dicht vor dem Trommelfell an der oberen Gehörgangswand, von welcher die Polypen zum Theil ausgingen, auf ranhen Knochen. Wenige Tage nach dieser Operation entstand, nachdem das Ohr inzwischen regelmässig mit Borsäurelösung ausgespritzt worden war, ein eigenthiimliches Fieber, das durch die nebenstehende Temperaturcurve illustrirt wird.

Schon am 26. August häufiges Frösteln; daneben Ohrenschmerzen, die nach dem Scheitel hinaufziehen. Eiterung gering, nicht uibelriechend. In der Tiefe des Gehörgangs pulsirende Lichtreflexe. Ordination: Eisbeutel auf den Warzenfortsatz.

29. August. Die Schmerzen vorüber. Warzenfortsatz nur an der Insertion des M. sternocleido-mastoideus druckempfindlich, sonst nirgends, auch nicht einmal bei Percussion schmerzhaft. Beim Spritzen entleeren sich mehrmals bröcklige, cholesteatomähnliche Massen.

1. September. Morgens Sehuittelfrost, Zunge belegt, Appetitlosigkeit. Starkes pulsirendes Ohrensausen.

R Fl. a. O. (3) nicht verstanden, c per Knochenleitung von der Stirnmitte nach rechts gehört.

3. September. Abends Schüttelfrost.

5. September. In den letzten Tagen geringe Eiterung; Bröckel sind fast gar nicht mehr gekommen. Mit der etwas gekrümmten Sonde 


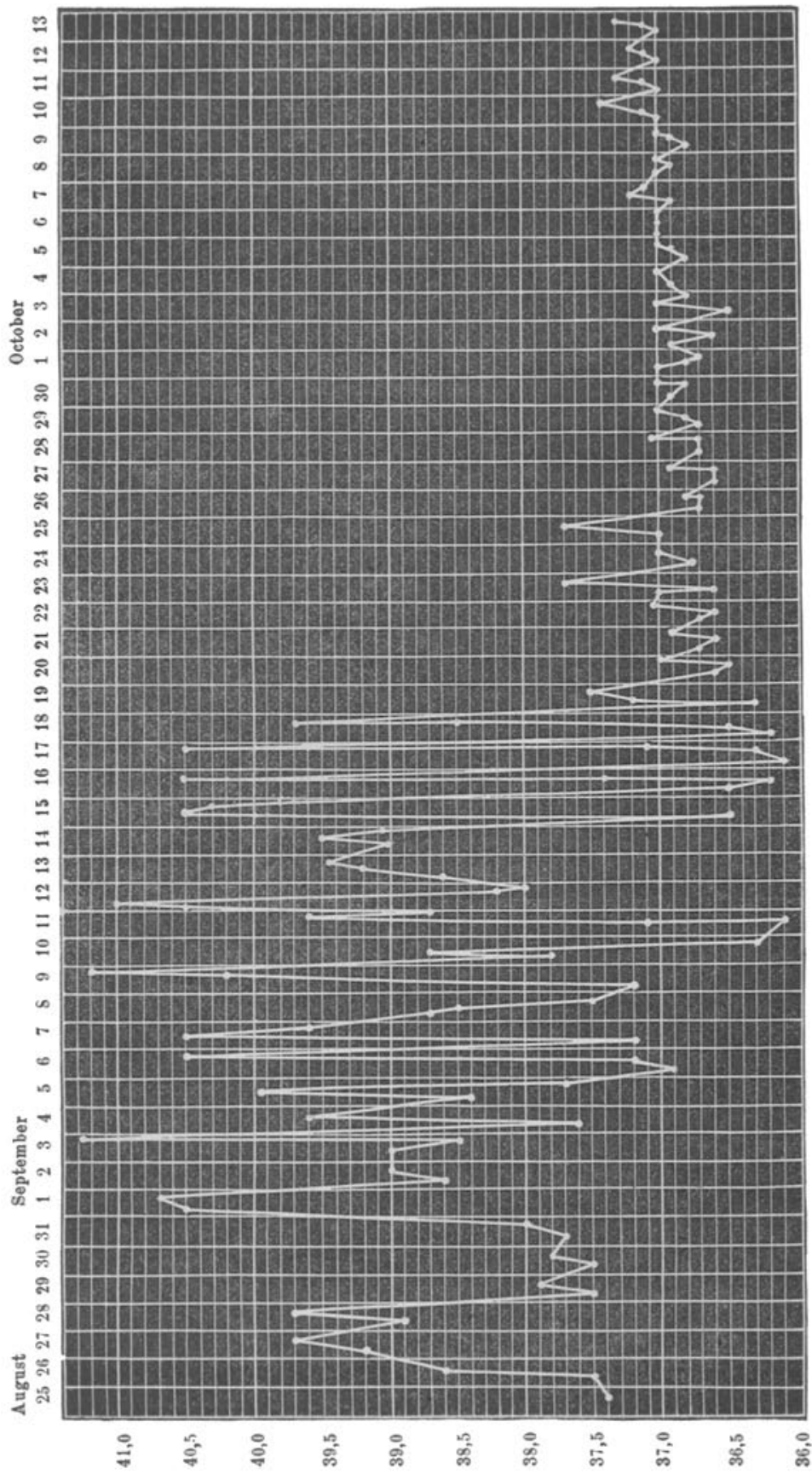


gelangt man von der vorderen oberen Gehörgangswand aus an cariösem Knochen vorbei in eine Höhle. In dieselbe wird heute ein $\mathrm{S}$-förmig gebogenes dünnes Bleirohr eingeführt und mittelst desselben Ausspritzungen vorgenommen. Hierbei entleeren sich zah lreiche Bröckel, und dann stürzt dicker gelber Eiter nach.

6. September. Abends Schüttelfrost.

7. September. Mittags Frost.

8. September. Beim Ausspritzen entleeren sich mehrere grössere Bröckel, wonach wieder dicker Eiter folgt.

9. September. Am Vormittag drei Frostanfälle. beseitigt wird.

10. September. Nachmittags Collaps, der aber durch Wein schnell

11. September. Morgens Frösteln. Beim Spritzen kommen Bröckel und hinterher eitriger Schleim.

12. September. Morgens zweimal Frösteln.

13. September. Morgens zweimal Frösteln. Ordination: Täglich Chinin. muriat. 0,8 Gim.

14. September. Mittags Frost.

15. September. Abends Frost.

16. September. Abends Frost.

17. September. Abends Frost.

19. September. Heute wird das Chinin ausgesetzt. Patientin fühlt sich viel wohler, hat guten Appetit. Bröekel haben sich in den letzten Tagen nicht mehr entleert. Secretion gering. Trommelfelle abgeblasst.

26. September. Das Befinden ist seit dem 19. andauernd gut geblieben. Warzenfortsatz auch am Ansatz des M. sternocleidomastoideus nicht mehr druckempfindlich. In der Membrana Shrapnelli eine ïber stecknadelkopfgrosse Perforation. Eiterung sehr gering. Ordination: Alcohol. absolut. zum Einträufeln ins Ohr.

16. October 1883. Entlassung aus der Klinik. Eiterung minimal. Trommelfell abgeblasst, etwas eingezogen.

R Fl. $8(3,8)$.

Patientin hat sich wieder ganz erholt.

Das nach der letzten Polypenoperation eingetretene Fieber zeigt den Charakter des pyämischen, raschen, regellosen Wechsel der Temperatur von den höchsten Erhebungen zu den tiefsten Abfällen, daneben häufig wiederholte, unregelmässig auftretende Schüttelfröste. Metastatische Entziindungen fehlten oder waren wenigstens nicht nachweisbar. Ob also hier eine Pyämie vorlag, mag dahingestellt bleiben, um so mehr, als die Definition dieser Erkrankung von verschiedenen Autoren noch in sehr differenter Weise gegeben wird.

Das Zustandekommen der schweren fieberhaften Entzündung im Anschluss an die operative Beseitigung der aus dem oberen Trommelhöhlenraum entspringenden polypösen Wucherungen er- 
kläre ich mir dadurch, dass nach Entfernung der letzteren das Spritzwasser ungehindert in die Tiefe dringen konnte und die in der Paukenhöhle vielleicht seit Jahren ruhenden Massen eingedickten Eiters zum Aufquellen brachte, wodurch einerseits purulente Entzündung, andererseits häufige Eiterretention bedingt werden musste. Durch sorgfältige Reinigung des Mittelohrs, wozu ein S-förmig gebogenes Röhrchen sich unumgänglich erforderlich erwies, gelang es uns, die aufgequollenen Massen allmählich herauszuspülen, dem durch sie zurïckgehaltenen Eiter Abfluss zu schaffen und schliesslich eine Heilung zu bewirken. Dass diese gründliche Entfernung der alten eingetrockneten Secretmassen aus dem Inneren des Schläfenbeins für die bleibende Gesundheit der Patientin von allergrösstem Vortheil ist, bedarf keiner besonderen Auseinandersetzung; wissen wir doch, dass von solchen Ansammlungen aus jederzeit plötzlich tödtliche Resorptionskrankheiten ihren Ausgang nehmen können.

Indem ich hiermit meine casuistischen Mittheilungen schliesse, gestatte ich mir noch mit wenigen Worten einiger Medicamente zu gedenken, welche wir in neuerer Zeit bei der Behandlung von Ohrenkranken sowohl in der Klinik, wie in der Poliklinik mit gutem Erfolge in Anwendung brachten.

In erster Reihe nenne ich die Aqua chlorata, welche sich uns bei Mittelohreiterungen vielfach als ein vortreffliches Mittel bewährt hat. Wir verordnen sie zu Instillationen in Form von Ohrtropfen unverdünnt, zu Ausspritzungen dagegen mit gleichen Theilen Wasser vermischt. Sie ist ein sehr energisches Desinficiens, daher namentlich bei fötider Otorrhoe von ansgezeichneter Wirksamkeit; ausserdem hat sie vor der Borsäurelösung den Vorzug, nicht nur die Secretion raseh zu beschränken, sondern auch kleinere Granulationen und Polypen zur Schrumpfung zu bringen. In chronischen Fällen wird sie meist, in acuten dagegen selten vertragen; in letzteren ist daher Borsäurelösung geeigneter.

Ein zweites Mittel, das ich erwähnen wollte, benutzen wir in denjenigen Fällen, in welchen in der Tiefe des Mittelohrs eingetrocknete Secretmassen oder cholesteatomartige Anhäufungen stagniren, zu Ausspritzungen. Es ist dieses eine Mis $\mathrm{eh}$ ung von Wasser und Alkohol zu gleichen Theilen; dieselbe empfieblt sich hier ganz vorzüglich, weil bei ihrer Anwendung ein Aufquellen der genannten Massen nicht stattfindet.

Berlin, im August 1884. 